\title{
Round-Robin Relaying with Diversity in Amplify-and-Forward Multi-Source Cooperative Communications
}

\author{
Zhang Zhang, Tiejun Lv, Senior Member, IEEE, and Shaoshi Yang, Student Member, IEEE
}

\begin{abstract}
In this paper, a round-robin based relay protocol dubbed round-robin relaying with source selection protocol (R3SSP) is proposed to achieve full cooperative diversity in multisource cooperative communication networks. In R3SSP, all the sources transmit their individual information in turn. The relays then forward the messages of some specific sources in a fixed order according to the limited feedback information. Compared with traditional relay selection based protocols, R3SSP is based on round-robin relaying, thus avoids relay selection and requires no specific channel state information (CSI) feedback. R3SSP can therefore be implemented with lower complexity. Furthermore, the exact and asymptotic expressions of the outage probability are derived. The diversity-multiplexing tradeoff (DMT) performance is also analyzed. Theoretical analysis shows that R3SSP achieves full cooperative diversity, and provides better DMT performance than relay selection based protocols in a system where the number of sources is higher than that of the relays. Based on the DMT analysis, we further propose an adaptive relay activation (ARA) scheme which is capable of achieving higher DMT by dynamically selecting the number of relays to be activated in the entire network. Simulation results also verify the validity and superiority of R3SSP.
\end{abstract}

Index Terms-Cooperative communication, round-robin relaying, diversity-multiplexing tradeoff (DMT), adaptive relay activation.

\section{INTRODUCTION}

$\mathbf{T}$ He use of diversity is considered as one of the most effective means to mitigate channel impairment caused by random fading in wireless environments [1]. Cooperative communication has emerged as a promising solution to achieve cooperative diversity in a distributed manner [2]. So far, a variety of cooperation methods have been introduced, for example the amplify-and-forward (AF) and the decode-andforward (DF) schemes. In a multi-relay cooperative network, a simple method to achieve full cooperative diversity is that

Copyright (c) 2012 IEEE. Personal use of this material is permitted. However, permission to use this material for any other purposes must be obtained from the IEEE by sending a request to pubs-permissions@ieee.org.

This work is financially supported by the National Natural Science Foundation of China (NSFC) (Grant No. 60972075 and 61271188). The paper was presented in part at the IEEE Global Communications Conference (GLOBECOM) 2011.

Z. Zhang and T. Lv are with the School of Information and Communication Engineering, Beijing University of Posts and Telecommunications (BUPT), Beijing 100876, China, (e-mail: zhangzhang86@bupt.edu.cn; lvtiejun@bupt.edu.cn).

S. Yang is with the School of Electronics and Computer Science, University of Southampton, SO17 1BJ Southampton, U.K., and also with the School of Information and Communication Engineering, Beijing University of Posts and Telecommunications (BUPT), Beijing 100876, China (e-mail: sy7g09@ecs.soton.ac.uk). all the relays assist the source to transmit in a round-robin fashion. However, the traditional round-robin relay protocol imposes considerable loss of spectral efficiency because each message from a single source is transmitted in multiple time slots. To solve this problem, strategies such as relay selection and space-time coded cooperation were exploited [2]-[8], [12]. Among these strategies, relay selection, which is also known as opportunistic relaying, attracts much attention. Protocols with relay selection do not rely on the assumption of precise time synchronization and are well-suited for both AF and DF systems. A range of relay selection algorithms with full cooperative diversity have been proposed [4]-[11], [30]-[32], and both centralized and distributed relay selection algorithms [7] can be employed in these protocols. However, specific channel state information (CSI) feedback is required in all relay selection based schemes, which increases the implementation complexity and decreases the transmission efficiency of the network.

On the other hand, some schemes have been recently proposed to increase the achievable throughput of a practical relay system which usually works in a half-duplex way [13][16], [24]-[27]. The basic idea of these works is to use fewer time slots for relaying by sharing a single relay time slot among different sources, which implies that a relay has the potential to assist the transmission of multiple sources simultaneously. However, these relay sharing schemes exploited network coding (NC) [19] to achieve throughput improvement, thus are more complicated and typically not applicable to AF relay systems.

For the purpose of dispensing with the specific CSI feedback, and inspired by the idea of relay sharing, in this paper, we propose a new relay protocol capable of achieving full cooperative diversity in AF systems. Observing that the operation of relay selection requires CSI feedback, we instead use all the relays in a round-robin fashion. To avoid the spectral efficiency loss which is caused by round-robin relaying, in this paper relay sharing is achieved by introducing source selection. Hence we dub the proposed protocol as round-robin relaying with source selection protocol (R3SSP). To be specific, unlike traditional time-resource allocation framework in which the relays serve one source immediately after the source's transmission, a new time-resource allocation framework is exploited, which includes two phases: the broadcast phase and the relay phase. In the broadcast phase, all the sources broadcast their individual information in turn. Then during the relay phase, all the relays help the transmission one by one. 
In each relay time slot, one relay provides diversity for all the sources by assisting the transmission of the "worst" source whose information most needs to be relayed. In this way, the "worst" source obtains retransmission diversity, whereas the other sources achieve selection diversity as a result of the source selection. The main contributions of this paper are listed as follows.

1) We investigate the system outage probability and the diversity-multiplexing tradeoff (DMT) performance of the proposed R3SSP. Our analysis demonstrates that R3SSP is capable of achieving full cooperative diversity and is superior to traditional relay selection based protocols in terms of the DMT performance when we have more sources than relays. It means that R3SSP is capable of improving the reliability/efficiency without loss of efficiency/reliability in certain scenarios. This conclusion is intriguing. By taking advantage of source selection, R3SSP makes the sources share the relays and hence greatly reduces the required time slots. To elaborate a little further, in the uplink of a centralized wireless network, such as the long term evolution (LTE) [22] and the worldwide interoperability for microwave access (WiMAX) [23] where the number of sources $N$ is usually larger than that of the relays $M$, R3SSP occupies totally $N+M$ time slots because all the sources share $M$ relay time slots. By comparison, the relay selection based traditional protocols occupy $2 N$ time slots. Therefore, the proposed R3SSP attains significant throughput improvement when there are more sources than relays, while maintaining the same level of reliability as its relay selection based counterparts do.

2) In contrast with traditional protocols that achieve full cooperative diversity with the aid of relay selection, R3SSP does not require CSI feedback to carry out source selection, rendering much convenience for practical implementation. The source selection is performed by means of the quality record of each source, which is simply characterized as the signal-to-noise ratio (SNR) of the combined signals of each source, and may be easily obtained by SNR estimation algorithms [20], [21] at the destination.

3) Based on the DMT analysis of R3SSP, an adaptive relay activation (ARA) strategy is then proposed to further improve the DMT performance of R3SSP. Noticing that activating all the relays is not always optimal in terms of DMT, we design an adaptive strategy that activates a proper number of relays in the whole system in light of the desired multiplexing gain. This ARA strategy can also be extended into the relay selection based schemes. However, R3SSP still outperforms the relay selection based schemes when ARA strategy is also exploited in all the benchmarking schemes concerned.

The rest of this paper is organized as follows. The motivation of our work and the system model are presented in Section II. The proposed R3SSP is described in Section III, followed by Section IV where the performance of R3SSP is analyzed. Then in Section V, the ARA scheme is proposed, and simulation results are provided in Section VI. Finally, conclusions are drawn in Section VII.

\section{Motivation And System Model}

\section{A. Motivation of Our Work}

Recently, some NC based relay schemes were also proposed for increasing the spectral efficiency by reducing the required number of time slots [13]-[16], [24]-[27]. In [13], [14], the authors proposed bit-wise soft NC schemes for fournode networks consisting of two sources, one relay and one destination. In [15], [16], [24]-[27], the authors focused on the usage of Galois field NC (GFNC) in multi-source DF relay networks. In these works, GFNC was exploited at the relay node for providing cooperative diversity to multiple sources. In [16], a three-time-slot transmission scheme for a four-node DF network containing two sources $\left(S_{1}\right.$ and $\left.S_{2}\right)$, one relay $(R)$ and one destination $(D)$ was proposed. This scheme is illustrated in Fig. 1(a), where $S_{1}$ and $S_{2}$ intend to send their respective symbols $x_{1}$ and $x_{2}$ to $D$. At first, $S_{1}$ and $S_{2}$ broadcast $x_{1}$ and $x_{2}$ to the relay $R$ and the destination $D$ in time-orthogonal channels, and $R$ decodes $x_{1}$ and $x_{2}$ as $\hat{x}_{1}$ and $\hat{x}_{2}$, respectively. Then $R$ employs GFNC and forwards $\hat{x}_{1} \oplus \hat{x}_{2}$ to $D$, where $\oplus$ denotes the bit-wise XOR operation. Both analysis and simulations show that this scheme provides a diversity order of two for both sources [16]. The authors of [24], [26], [27] studied more general network models. In [24], the authors extended the scheme of [16] to general multi-source single-relay networks and analyzed its error rate performance. In [26], the authors extended the network model and the scheme of [24] to a cooperative diversity scheme designed for wireless systems employing both NC as well as the combination of bit-interleaved coded modulation (BICM) and orthogonal frequency division multiplexing (OFDM). In [27], the authors investigated the design of the GFNC at the relay nodes of a two-source two-relay network from the perspective of linear block codes, and proposed several fourtime-slot communication schemes. Inspired by these works, in [25] the authors studied the GFNC design for general multisource multi-relay networks. These studies lead to a conclusion that the DF system can exploit GFNC to achieve the "relay sharing" effect, which means multiple sources are able to benefit from one single relay time slot. However, these works either focus on some special networks [13]-[16], [24], [26], [27], or fail to achieve full cooperative diversity [25], [27]. Furthermore, GFNC is not applicable to AF systems because AF relays have no decoding capability. Therefore, the protocol design for a general multi-source multi-relay network remains an open problem, especially for AF networks.

In this paper, we will show that AF systems are also capable of achieving "relay sharing" effect with the aid of a welldesigned protocol. The reason is that in essence the "relay sharing" effect comes from selection, and GFNC constitutes one of the tools to carry out selection. From the perspective of linear block codes, GFNC provides redundancy to all the original data for ensuring that the incorrect decoding of some encoded data would not affect the correctness of the whole original data. Consider the scheme in [16] as an example, the 


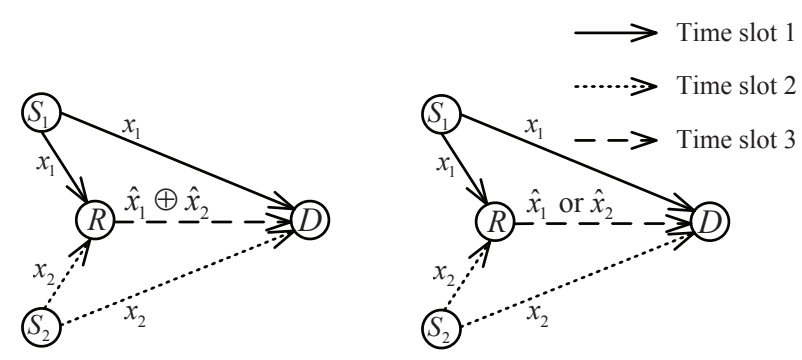

(a) GFNC based relaying for DF

(b) Selection based relaying for $\mathrm{AF}$

Fig. 1. An example to improve spectrum efficiency in a four-node network.

original symbols $x_{1}, x_{2}$ are encoded to $x_{1}, x_{2}$, and $\hat{x}_{1} \oplus \hat{x}_{2}$. If $D$ decodes any one of $x_{1}, x_{2}, \hat{x}_{1} \oplus \hat{x}_{2}$ in error, it can still correctly recover $x_{1}, x_{2}$ with the aid of the other two received symbols. All $D$ has to do is to pick out the most unreliable symbol and to decode $x_{1}, x_{2}$ with the two most reliable symbols. Therefore, potentially, other operations instead of GFNC may be exploited in AF systems to attain "relay sharing" effect as long as the right selection can be made. To gain more insights into the above mentioned conclusion, in this paper we propose a source-selection based relay scheme which is suitable for both AF and DF systems. The proposed scheme is exemplified in Fig. 1(b). After receiving $x_{1}$ and $x_{2}$ from direct links, $D$ determines which received symbol is more unreliable and calls $R$ to retransmit the selected symbol. Then $R$ sends its observation of the selected symbol to $D$. It can be proved that in this scheme both $S_{1}$ and $S_{2}$ achieve a diversity order of two. We are interested in the fundamental mechanism how both sources obtain the diversity order of two. To elucidate this point, we give an intuitive explanation here. Without loss of generality, let us suppose $x_{1}$ is selected to be relayed. Obviously, since $D$ receives two copies of $x_{1}$, $x_{1}$ obtains a diversity order of two through retransmission. On the other hand, since $x_{2}$ is a more reliable sample out of the two independent direct transmissions of $x_{1}$ and $x_{2}, x_{2}$ is supposed to be successfully recovered unless both samples from the independent direct links are decoded in error (i.e., when $\hat{x}_{1}$ and $\hat{x}_{2}$ are both erroneous). As a result, albeit being transmitted only once, $x_{2}$ also enjoys a diversity order of two. We contend that this diversity gain comes from selection operation. It implies that the unselected sample gets selection diversity.

\section{B. System Model}

We consider a network with $N+M+1$ nodes, where $N$ sources $\left(S_{n}, 1 \leq n \leq N\right)$ transmit individual information to one destination $(D)$ with the aid of $M$ relays $\left(R_{m}, 1 \leq\right.$ $m \leq M$ ). In the uplink of wireless communication systems such as LTE and WiMAX, it is reasonable to assume that $N \geq M$. The system model is shown in Fig. 2. All the nodes are assumed to have single antenna and transmit with power $E_{s}$, and to operate in half-duplex mode. All the channels $\left(S_{n} \rightarrow R_{m}, S_{n} \rightarrow D, R_{m} \rightarrow D^{1}\right)$ in the network are

\footnotetext{
${ }^{1}$ The notation $A \rightarrow B$ represents a link from node $A$ to node $B$.
}

assumed to be independent Rayleigh block fading channels with additive white Gaussian noise (AWGN). It means that the channel coefficients remain fixed during each time slot and change independently among different time slots.

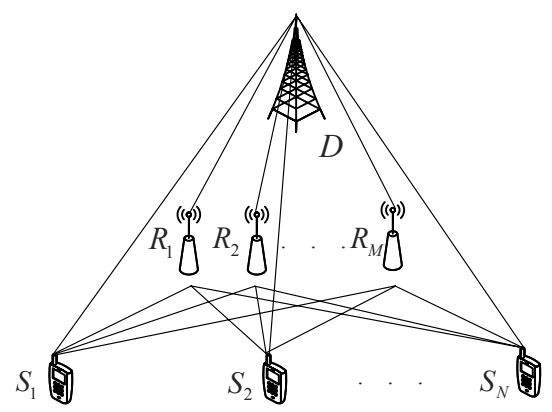

Fig. 2. System Model.

\section{BASIC PROTOCOL DESCRIPTION}

The whole transmission is divided into two phases: the broadcast phase and the relay phase. First, all the sources transmit in a round-robin manner in the broadcast phase. Hence the broadcast phase occupies $N$ time slots. Then in the relay phase, the relays assist source transmissions one by one, and the relay phase lasts for $M$ time slots. Fig. 3(a) illustrates the time resource allocation.

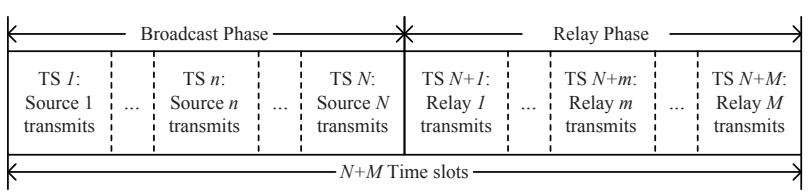

(a)

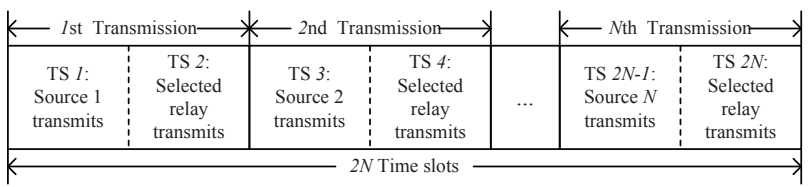

(b)

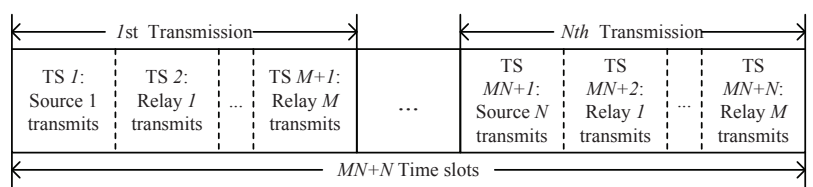

(c)

Fig. 3. Time-resource allocation for (a) R3SSP, (b) relay selection based protocols and (c) traditional round-robin protocols.

\section{A. Broadcast Phase}

In the broadcast phase, each source broadcasts its data in turn. For the source $S_{n}$, the received signals at relay $R_{m}$ and the destination $D$ are

$$
\begin{aligned}
y_{S_{n} R_{m}} & =h_{S_{n} R_{m}} x_{n}+n_{S_{n} R_{m}}, \\
y_{S_{n} D} & =h_{S_{n} D} x_{n}+n_{S_{n} D},
\end{aligned}
$$

respectively, where $h_{S_{n} R_{m}}$ and $h_{S_{n} D}$ are the fading coefficients of the links $S_{n} \rightarrow R_{m}$ and $S_{n} \rightarrow D$ in the $n$th time 
slot of the broadcast phase, respectively. $h_{S_{n} R_{m}}$ and $h_{S_{n} D}$ are modeled as independent zero-mean circularly symmetric complex Gaussian (ZMCSCG) random variables (RVs) with variances $\gamma_{S_{n} R_{m}}$ and $\gamma_{S_{n} D}$, respectively. $x_{n}$ is the transmitted symbol of $S_{n} \cdot n_{S_{n} R_{m}}$ and $n_{S_{n} D}$ denote the AWGN with zero mean and variance $N_{0}$. After each source's transmission, $D$ initializes the SNR of the signal of each source by estimating the SNR of the corresponding received signal. Denote $\xi_{S_{n} D}$ as the SNR of the received signal at $D$ from $S_{n}$ in the broadcast phase. $\xi_{S_{n} D}$ may be written as

$$
\xi_{S_{n} D}=\frac{E_{s}\left|h_{S_{n} D}\right|^{2}}{N_{0}},
$$

where $E_{s}$ is the average transmit power in each node.

\section{B. Relay Phase}

The relay phase lasts for $M$ time slots, in which all the relays participate in transmission one by one, i.e., in a roundrobin fashion. For example, in the first relay time slot, $R_{1}$ transmits, then in the second relay time slot, $R_{2}$ transmits, and this procedure goes on until all the relays have assisted the transmission. Briefly speaking, the relays assist the multisource transmissions with round-robin scheduling. In each time slot, the "worst" source is selected according to the quality records (i.e., the SNRs of the combined signals of all the sources) stored at $D$, and one relay in operation amplifies the received signal from the selected source and forwards the signal to $D$. Upon receiving the relayed signal, $D$ combines all the received signals related to the selected source and then updates the quality record of each source to prepare for the next relay time slot. The combined signals are transmitted from different nodes in different time slot, and thus improves diversity performance. A variety of data combining schemes can be exploited, such as maximum ratio combining (MRC) [28], equal gain combining (EGC) and selective combining (SC). Since $\mathrm{SC}$ is able to yield diversity with rather low complexity, we focus on $\mathrm{SC}$ in this paper, while the other combining schemes can also be invoked by R3SSP straightforwardly. The details of R3SSP are presented as follows.

We denote $\xi_{n}^{(m)}$ as the SNR of the combined signals of $S_{n}$ recorded at $D$ before the $m$ th relay time slot, and $S N R_{m}$ as the set $\left\{\xi_{n}^{(m)} \mid 1 \leq n \leq N\right\}$. The quality records of the sources stored at $D$ can be characterized by $\xi_{n}^{(m)}$. For the ease of exposition, the detailed calculation and updating of $\xi_{n}^{(m)}$ will be explained later.

In the $m$ th relay time slot, $D$ first selects the "worst" source and broadcasts its index number $\theta_{m},{ }^{2}$ where

$$
\theta_{m}=\arg \min _{n=1, \ldots, N} \xi_{n}^{(m)}, 1 \leq m \leq M .
$$

It should be noted that one source can be selected multiple times during the whole relay phase. In other words, if one source is selected as the "worst" source in one relay time slot, the relay has to forward its observation of the signal from this source regardless of the fact whether this source has been

\footnotetext{
${ }^{2}$ This limited feedback information occupies $\left\lceil\log _{2} N\right\rceil$ bits.
}

selected in previous relay time slots or not. Then $R_{m}$ amplifies its observation of the signal from $S_{\theta_{m}}$ and forwards it to $D$. $D$ receives the relayed signal of

$$
y_{R_{m} D}=h_{R_{m} D} \widetilde{x}_{\theta_{m}}+n_{R_{m} D}
$$

where $h_{R_{m} D}$ is a ZMCSCG RV with parameter $\gamma_{R_{m} D}$ and represents the channel fading coefficient of the link $R_{m} \rightarrow D$ in the $m$ th time slot of the relay phase, and $n_{R_{m} D}$ is the AWGN with zero mean and variance $N_{0}$, while $\widetilde{x}_{\theta_{m}}$ is the amplified signal of $x_{\theta_{m}}$ at $R_{m}$ and is expressed as

$$
\widetilde{x}_{\theta_{m}}=\frac{\left|h_{S_{\theta_{m}} R_{m}}\right|}{\sqrt{\left|h_{S_{\theta_{m}} R_{m}}\right|^{2}+N_{0}}} x_{\theta_{m}}+\frac{n_{S_{\theta_{m}} R_{m}}}{\sqrt{\left|h_{S_{\theta_{m}} R_{m}}\right|^{2}+N_{0}}} \text {. }
$$

After $R_{m}$ finishes the transmission, $D$ employs $\mathrm{SC}$ to perform data combining. Finally, $D$ updates $\xi_{\theta_{m}}^{(m)}$ to $\xi_{\theta_{m}}^{(m+1)}$ and reconstructs a new SNR set $S N R_{m+1}$ to record all the sources' quality of the next relay time slot, and the current $m$ th relay time slots ends.

The relay phase continues until all the relays finish assisting transmission and thereby lasts for $M$ time slots.

To be more specific, the calculation and updating of $\xi_{n}^{(m)}$ are described as follows.

In each relay time slot, $D$ decides which source's message should be relayed in the next relay's transmission according to each source's quality record $\xi_{n}^{(m)}$, then performs data combining and renews $\xi_{n}^{(m)}$ to $\xi_{n}^{(m+1)}$.

For the initialization, $\xi_{n}^{(1)}$ is the SNR of the received signals at $D$ from $S_{n}$ after the broadcast phase, and obviously it is equal to $\xi_{S_{n} D}$ which is expressed in (3).

In the $m$ th relay time slot, from (6), the SNR of the received signal at $D$ (i.e., the SNR of $y_{R_{m} D}$ ) is

$$
\begin{aligned}
\xi_{S_{\theta_{m}} R_{m} D} & =\frac{E_{s}\left|h_{S_{\theta_{m}} R_{m}} h_{R_{m} D}\right|^{2}}{N_{0}\left(\left|h_{S_{\theta_{m}} R_{m}}\right|^{2}+\left|h_{R_{m} D}\right|^{2}+N_{0}\right)} \\
& =\frac{\xi_{S_{\theta_{m}} R_{m}} \xi_{R_{m} D}}{\xi_{S_{\theta_{m}} R_{m}}+\xi_{R_{m} D}+1},
\end{aligned}
$$

where $\xi_{S_{\theta_{m}} R_{m}}$ is the SNR of the link $S_{\theta_{m}} \rightarrow R_{m}$ in the $m$ th broadcast time slot and can be expressed as $\xi_{S_{\theta_{m}} R_{m}}=$ $E_{s}\left|h_{S_{\theta_{m}} R_{m}}\right|^{2} / N_{0}, \xi_{R_{m} D}$ is the SNR of the link $R_{m} \rightarrow D$ in the $m$ th relay time slot and can be expressed as $\xi_{R_{m} D}=$ $E_{s}\left|h_{R_{m} D}\right|^{2} / N_{0}$.

After data combining, $\xi_{\theta_{m}}^{(m)}$ is updated to $\xi_{\theta_{m}}^{(m+1)} \cdot \xi_{\theta_{m}}^{(m+1)}$ is the SNR of the combined signal at the output of the selection combiner, thus it is written as

$$
\xi_{\theta_{m}}^{(m+1)}=\max \left\{\xi_{\theta_{m}}^{(m)}, \xi_{S_{\theta_{m}} R_{m} D}\right\} .
$$

Since only the signal of the selected source is relayed, the unchosen sources' quality records remain unchanged. Thus the elements in the updated SNR set $S N R_{m+1}$ are

$$
\xi_{n}^{(m+1)}=\left\{\begin{array}{ll}
\xi_{n}^{(m)}, & n \neq \theta_{m} \\
\max \left\{\xi_{n}^{(m)}, \xi_{S_{\theta_{m}} R_{m} D}\right\}, & n=\theta_{m}
\end{array} .\right.
$$

It should be noted that R3SSP can be easily extended into DF systems with minor changes in data combining, SNR 
calculation and updating, because the SNR expressions of the relayed signals in DF systems are different from their counterparts in AF systems.

\section{Comparisons with traditional protocols}

Below we will compare R3SSP with traditional protocols that use relay selection and round-robin relaying. Fig. 3 demonstrates the time-resource allocation of R3SSP and the traditional protocols. The total numbers of time slots of R3SSP and relay selection protocols are $N+M$ and $2 N$, respectively, whereas that of traditional round-robin relaying protocol is $(M+1) N$. Compared with traditional round-robin relaying protocol, R3SSP and relay selection based protocols greatly improve spectral efficiency by the selection operation. In addition, R3SSP requires less time resources than relay selection based protocols, thereby it has potential to provide higher data rate in wireless networks where there are more sources than relays. To show the superiority of R3SSP, in the next section we will prove that R3SSP is able to achieve full cooperative diversity and can yield a better DMT performance when compared with traditional round-robin and relay selection protocols.

Moreover, the complexity comparison between R3SSP and its relay selection based counterparts is performed. To be specific, in each relay time slot of relay selection based protocols, $D$ selects the best relay from all the $M$ relays, thus the complexity of relay selection operation per relay time slot is $\mathcal{O}(M)$. There are $N$ relay time slots in relay selection based protocols, hence the total selection complexity during the whole transmission is $\mathcal{O}(N M)$. In contrast, as far as R3SSP is concerned, $D$ selects the "worst" source from all the $N$ sources in each relay time slot. Therefore, the complexity of source selection operation per relay time slot is $\mathcal{O}(N)$. Since there are $M$ relay time slots in R3SSP, the total selection complexity of the whole transmission is also $\mathcal{O}(N M)$. Therefore, both the relay selection based protocols and the proposed R3SSP have the same level of complexity with respect to the selection algorithm.

Another important issue is to compare the degree of difficulty for obtaining the required CSI in each scheme. Specifically, in relay selection based schemes, global CSI is required to provide full cooperative diversity. Centralized and distributed schemes [7], [8] have been proposed to carry out relay selection when using relay selection based protocols [4]-[11]. In the centralized relay selection scheme, a central controller (i.e., $D$ ) conducts the selection operation. The central controller has to know the global CSI before it makes the decision on relay selection, which includes all the channels between the sources and the relays. That is to say, $D$ has to know the instantaneous CSI of the links irrelevant to it. This CSI can only be obtained via CSI feedback. To get rid of the demand of global CSI, the distributed relay selection scheme uses the method of distributed timers to perform selection. To elaborate a little further, each relay gets the instantaneous CSI of the link from itself to $D$, and then starts a timer whose duration is inversely proportional to a metric that depends only on the quality of its received signals and on the quality of the channel from itself to $D$. Because the timer of the "best" relay has the shortest duration, the "best" relay starts transmission first. The other relays stop their timers as soon as they detect the "best" relay's signal (readers interested in details of this approach are suggested to refer to [7], [8]). Indeed, the distributed relay selection scheme reduces the implementation complexity. However, each relay has to know the instantaneous CSI of the link from itself to $D$. It means that the CSI of the link is required at the transmitter side, thus CSI feedback still cannot be omitted. Furthermore, since all the relays compete for transmission according to their own channel conditions, there is a possibility of collision that multiple relays access the wireless medium simultaneously [7], [8]. On the other hand, if each relay could not listen to each other, it would be more complex to perform the selection task in the distributed relay selection scheme (please refer to [8] for the details). In summary, specific CSI feedback between the relays and the destination are required in both the centralized and the distributed schemes for selecting the "best" relay. This requirement restricts the applications of relay selection based protocols in practice. In contrast with relay selection protocols, R3SSP works in a round-robin manner, thus avoids relay selection operation. R3SSP makes source selection according to the quality records of all the received signals at $D$. Note that $D$ calculates the quality record of each source according to the SNRs of its already received signals, which can be easily obtained by using SNR estimation. Therefore, we contend that there is no requirement of CSI feedback in R3SSP. $D$ only has to notify the relays of the index number of the selected source with a short broadcast message.

\section{Performance Analysis}

In this section, we study the performance of the proposed scheme in terms of outage behavior and DMT performance. The outage behavior is derived in both exact and approximate expressions. In order to provide a comprehensive insight into the proposed R3SSP, the DMT of the proposed scheme is investigated to show both its diversity and multiplexing performance in the high SNR regime.

For sake of clarity, the following definitions are made before starting analysis.

\section{A. Definitions}

$\lambda_{S_{n} R_{m}}, \lambda_{S_{n} D}$ and $\lambda_{R_{m} D}$ are defined as the average SNRs of the link $S_{n} \rightarrow R_{m}, S_{n} \rightarrow D$ and $R_{m} \rightarrow D$, respectively. They can be expressed as $\frac{N_{0}}{E_{s} \gamma_{S_{n} R_{m}}}, \frac{N_{0}}{E_{s} \gamma_{S_{n} D}}$ and $\frac{N_{0}}{E_{s} \gamma_{R_{m} D}}$, respectively, where $\gamma_{S_{n} R_{m}}, \gamma_{S_{n} D}$ and $\gamma_{R_{m} D}$ are expectations of $\left|h_{S_{n} R_{m}}\right|^{2},\left|h_{S_{n} D}\right|^{2}$ and $\left|h_{R_{m} D}\right|^{2}$, respectively. Thus the SNRs of all the links $S_{n} \rightarrow R_{m}, S_{n} \rightarrow D$ and $R_{m} \rightarrow D$ are RVs following exponential distributions with parameters $\lambda_{S_{n} R_{m}}, \lambda_{S_{n} D}$ and $\lambda_{R_{m} D}$, respectively.

Define $\mathrm{U}=\left\{u_{1}, \ldots, u_{k}, \ldots, u_{N+M}\right\}$ as the set of SNRs of the received signals at $D$ in the broadcast phase and the relay phase. More specifically, $u_{k}$ is defined as the SNR of the $k$ th broadcast time slot if $k \leq N$, and the SNR of the $(k-N)$ th relay time slot if $k>N$. Considering (3), (7) and (9), $u_{k}$ is 
written as

$$
u_{k}= \begin{cases}\xi_{k}^{(1)}, & 1 \leq k \leq N \\ \xi_{S_{\theta_{k-N}} R_{k-N} D}, & N<k \leq N+M\end{cases}
$$

We assume the elements of the set $U$ are ordered as $u_{1}^{\prime} \leq$ $u_{2}^{\prime} \leq \ldots \leq u_{N+M}^{\prime}$.

Based on (10), $\xi_{n}^{(M+1)}$ represents the SNR of the combined signals of $S_{n}$ at $D$ after the whole transmission. For the sake of clarify, we denote $\xi_{n}^{(M+1)}$ as $v_{n}$, i.e.,

$$
v_{n}=\xi_{n}^{(M+1)}
$$

We then define $\mathrm{V}$ as the set of $v_{n}$, i.e., $\mathrm{V}=$ $\left\{v_{n} \mid n=1,2, \ldots, N\right\}$, whose elements are assumed to be ordered as $v_{1}^{\prime} \leq v_{2}^{\prime} \leq \ldots \leq v_{N}^{\prime}$.

An ordering $\left(\psi_{1}, \ldots, \psi_{N+M}\right)$ of $(1, \ldots, N+M)$ is called a source-selection ordering if $u_{k}^{\prime}=u_{\psi_{k}}$ for any $1 \leq k \leq$ $N+M$. It means that the $k$ th smallest SNR of all the received signals at $D$ during the whole transmission is $u_{\psi_{k}}$. All the possible source-selection orderings compose the ordering set $\Psi$.

The vector $\left(\theta_{1}, \ldots, \theta_{M}\right)$ is called a source-selection result where $\theta_{m}$ is defined as (4). It is readily verified that there exists and only exists one source-selection result corresponding to any given source-selection ordering.

The average transmit SNR of the network is defined as

$$
\rho=\frac{E_{b}}{N_{0}}=\frac{N E_{s}}{(N+M) N_{0}}
$$

where $E_{b}$ is the normalized average transmit power in the network. It should be mentioned that the specific modulation constellation is not considered in (13).

\section{B. System Outage Probability}

A system outage event occurs when $D$ is not able to correctly decode all the sources' information. Denote $I_{n}$ as the maximum average mutual information between $S_{n}$ and $D$. For a given end-to-end data rate of $\mathcal{R}$ bit/s/Hz, $S_{n}$ suffers an outage if $I_{n}=\frac{N}{N+M} \log \left(1+v_{n}\right)<\mathcal{R}$. Thus the system outage takes place if the condition $\min \left\{I_{1}, I_{2}, \ldots, I_{N}\right\} \geq \mathcal{R}$ is not satisfied.

By the definition of the system outage event, the system outage probability is expressed as

$$
\begin{aligned}
P_{\text {out }}(\mathcal{R}) & =1-\operatorname{Pr}\left(\min \left\{I_{1}, I_{2}, \ldots, I_{N}\right\} \geq \mathcal{R}\right) \\
& =1-\operatorname{Pr}\left(\min \left\{v_{1}, v_{2}, \ldots, v_{N}\right\} \geq 2^{\frac{N+M}{N} \mathcal{R}}-1\right) \\
& =1-\operatorname{Pr}\left(v_{1}^{\prime} \geq 2^{\frac{N+M}{N}} \mathcal{R}-1\right) \\
& =\mathrm{F}_{v_{1}^{\prime}}(\beta)
\end{aligned}
$$

where $\mathrm{F}_{v_{1}^{\prime}}($.$) is the cumulative distribution function (CDF) of$ $v_{1}^{\prime}, \beta=2^{\frac{N+M}{N}} \mathcal{R}-1$.

To begin with, we study the equivalent SNR of the combined signal at $D$ for each source after the whole transmission. As described in Section III, there are $N+M$ signals transmitted during the whole transmission. Since one signal is discarded while making data combining in each relay time slot, $N$ signals are kept for the final decision. We proceed to reveal that the most "useful" signals are selected for the final decision, i.e., in R3SSP, each "selected" signal (the received signals kept for the final decision of each source's information) has higher quality than any discarded signals. Obviously, all the elements in $U$ are independent RVs. Based on (10) and the definition of $\xi_{n}^{(m)}$, we readily have $\xi_{n}^{(m)} \in \mathrm{U}$ for all $n$ and $m(1 \leq n \leq N$ and $1 \leq m \leq M)$ and $\mathrm{V} \subseteq \mathrm{U}$. From (4) and (10), we have $v_{n}=\xi_{n}^{(M+1)} \geq \xi_{n}^{(m+1)}=\xi_{n}^{(m)}>\xi_{\theta_{m}}^{(m)}$ if $n \neq \theta_{m}$. It means that whenever $S_{n}$ is not selected in one relay time slot, we can find an element $\xi_{\theta_{m}}^{(m)} \in \mathrm{U}$ that is less than $v_{n}$. Similarly, we obtain $v_{n}=\xi_{n}^{(M+1)} \geq \xi_{n}^{(m+1)}>\min \left\{\xi_{\theta_{m}}^{(m)}, \xi_{S_{\theta_{m}} R_{m} D}\right\}$ if $n=\theta_{m}$. It means that once $S_{n}$ is selected in the relay phase, an element $\min \left\{\xi_{\theta_{m}}^{(m)}, \xi_{S_{\theta_{m}} R_{m} D}\right\} \in \mathrm{U}$ is found to be less than $v_{n}$. In summary, after each relay time slot, we can always find an element in $\mathrm{U}$ which is less than $v_{n}$. Therefore, after the whole relay phase, there are at least $M$ elements in $\mathrm{U}$ which are less than $v_{n}$, i.e., $v_{n}^{\prime} \geq u_{n+M}^{\prime}$. On the other hand, note that $\mathrm{V} \subseteq \mathrm{U}$, we have $v_{n}^{\prime} \leq u_{n+M}^{\prime}$. Therefore, we have

$$
v_{n}^{\prime}=u_{n+M}^{\prime},
$$

which indicates that $v_{n}^{\prime}$ is the $(M+n)$ th smallest SNR of all the received signals during the whole transmission. Thus (14) can be rewritten as

$$
P_{\text {out }}(\mathcal{R})=\mathrm{F}_{u_{M+1}^{\prime}}(\beta),
$$

where $\mathrm{F}_{u_{M+1}^{\prime}}($.$) is the CDF of u_{M+1}^{\prime}$.

1) Symmetric Network: First, we analyze the outage behavior and diversity order of R3SSP in symmetric networks. A symmetric network is a network where $\lambda_{S_{1} R_{m}}=\ldots=$ $\lambda_{S_{n} R_{m}}=\ldots=\lambda_{S_{N} R_{m}}$ for all $n$ and any fixed $m$. Hence we can denote $\lambda_{S_{n} R_{m}}$ as $\lambda_{S R_{m}}=\frac{N_{0}}{E_{s} \gamma_{S R_{m}}}$ in symmetric networks. Note that in a symmetric network, the distribution of $u_{k}$ is irrelevant to the source-selection ordering, i.e., no matter which source is selected at each relay time slot, the distribution of $u_{k}$ remains the same. Define $\mathrm{F}_{u_{k}}($.$) as the CDF of u_{k}$, in a symmetric network, when $1 \leq k \leq N, u_{k}$ is the SNR of the direct link $S_{k} \rightarrow D$ and its $\mathrm{CDF}$ is given as

$$
\mathrm{F}_{u_{k}}(x)=1-\exp \left(-\lambda_{S_{k} D} x\right) .
$$

When $N+1 \leq k \leq N+M, u_{k}$ is the SNR of the two-hop link $S_{\theta_{k-N}} \rightarrow R_{k-N} \rightarrow D$ and its CDF is [17]

$$
\begin{aligned}
\mathrm{F}_{u_{k}}(x)= & -\exp \left(-\lambda_{S R_{m}} x-\lambda_{R_{m} D} x\right) \\
& \times 2 \sqrt{\lambda_{S R_{m}} \lambda_{R_{m} D} x(x+1)} \\
& \times K_{1}\left(2 \sqrt{\lambda_{S R_{m}} \lambda_{R_{m} D} x(x+1)}\right),
\end{aligned}
$$

where $K_{1}($.$) is the first order modified Bessel function of the$ second kind.

According to the Total Probability Theorem and (16), 
$\mathrm{F}_{u_{M+1}^{\prime}}(\beta)$ can be written as

$\mathrm{F}_{u_{M+1}^{\prime}}(\beta)$

$=\operatorname{Pr}($ at least $M+1$ entries of $\mathrm{U}$ are less than or equal to $\beta$ )

$=\sum_{l=M+1}^{N+M} \operatorname{Pr}($ exactly $l$ entries of $\mathrm{U}$ are less than or equal to $\beta)$

Let us assume $j_{1}<\ldots<j_{l}$ are the indices of the $l$ smallest elements in $\mathrm{U}$, and $j_{l+1}<\ldots<j_{N+M}$ are the indices of the $N+M-l$ largest elements in $\mathrm{U}$. For given $j_{1}, \ldots, j_{N+M}$, we have

$$
\begin{aligned}
& \operatorname{Pr}\left(u_{j_{1}}, \ldots, u_{j_{l}} \leq \beta<u_{j_{l+1}}, \ldots, u_{j_{N+M}}\right) \\
= & \prod_{i=1}^{l} \mathrm{~F}_{u_{j_{i}}}(\beta) \prod_{i=l+1}^{N+M}\left(1-\mathrm{F}_{u_{j_{i}}}(\beta)\right) .
\end{aligned}
$$

The probability that exactly $l$ entries of $U$ are less than or equal to $\beta$ can be expressed as the sum of the probabilities $\operatorname{Pr}\left(u_{j_{1}}, \ldots, u_{j_{l}} \leq \beta<u_{j_{l+1}}, \ldots, u_{j_{N+M}}\right)$ over all the possible $j_{1}, \ldots, j_{N+M}$. Therefore, (19) is calculated as

$$
\begin{aligned}
& \mathrm{F}_{u_{M+1}^{\prime}}(\beta) \\
= & \sum_{l=M+1}^{N+M} \sum_{\substack{\text { all the possible } \\
j_{1}, \ldots, j_{N+M}}} \operatorname{Pr}\left(u_{j_{1}}, \ldots, u_{j_{l}} \leq \beta<u_{j_{l+1}}, \ldots, u_{j_{N+M}}\right) \\
= & \sum_{l=M+1}^{N+M} \sum_{\substack{1 \leq j_{1} \ldots<j_{l} \leq N+M \\
1 \leq j_{l+1} \ldots<j_{N+M} \leq N+M \\
j_{1} \neq j_{2} \neq \ldots \neq j_{N+M}}}^{l} \prod_{i=1}^{l} \mathrm{~F}_{u_{j_{i}}}(\beta) \prod_{i=l+1}^{N+M}\left(1-\mathrm{F}_{u_{j_{i}}}(\beta)\right) .
\end{aligned}
$$

Substituting (17) and (18) into (21), the closed form of the outage probability is obtained. To give insights into the diversity order of the proposed R3SSP, we investigate the asymptotic performance in the high SNR regime. As the SNR of each link goes to infinity, $\lambda_{S D}, \lambda_{S R}$ and $\lambda_{R D}$ approach zero. ${ }^{3}$ With the aid of the approximations that $\exp (t) \approx 1-t$ and $K_{1}(t) \approx \frac{1}{t}$ when $t \rightarrow 0,(21)$ is further formulated as

$$
\begin{aligned}
& \sum_{i=1}^{\mathrm{F}_{u_{M+1}^{\prime}}(\beta)} \sum_{\substack{1 \leq j_{1} \ldots<j_{i} \leq M \\
1 \leq j_{i+1} \cdots<j_{M+1} \leq N}}^{M} \prod_{t=1}^{i} \beta\left(\lambda_{S R_{j_{t}}}+\lambda_{R_{j_{t}} D}\right) \prod_{t=i+1}^{M+1} \beta \lambda_{S_{j_{t} D}} \\
= & \sum_{i=1}^{M} \sum_{\substack{1 \leq j_{1} \ldots<j_{i} \leq M \\
1 \leq j_{i+1} \cdots<j_{M+1} \leq N}}\left(\frac{N_{0}}{E_{b}}\right)^{M+1}\left(\frac{N \beta}{N+M}\right)^{M+1} \\
& \times \prod_{t=1}^{i} \frac{\gamma_{S R_{j_{t}}}+\gamma_{R_{j_{t}} D}}{\gamma_{S R_{j_{t}}} \gamma_{R_{j_{t}} D}} \prod_{t=i+1}^{M+1} \frac{1}{\gamma_{S_{j_{t}} D}} \\
\propto & \left(\frac{N_{0}}{E_{b}}\right)^{M+1} .
\end{aligned}
$$

\footnotetext{
${ }^{3}$ It should be noted that this conclusion holds subject to the condition that $\lim _{\rho \rightarrow \infty} \frac{N \beta}{\rho(N+M)}=0$, where $\beta$ is a constant and this condition is satisfied for any given fixed data rate.
}

It can be observed from (23) that the proposed R3SSP provides a diversity order of $M+1$ in a symmetric network. This conclusion agrees with the intuition that the diversity order of R3SSP is supposed to be $M+1$ because the most "useless" $M$ samples are discarded.

2) Asymmetric Network: Different from the symmetric networks, in the general asymmetric networks where the distributions of SNRs from different source-to-relay links are characterized by different parameters, the distribution of $u_{N+m}$ is also varying corresponding to the distinct source-selection orderings. Note that for a given source-selection orderings, $u_{N+m}$ obeys a distribution having deterministic parameters. In this case, the calculation of the system outage probability is very complicated, and the analysis and calculation procedure are detailed in Appendix A. For the sake of simplicity, an alternative approach is that we can construct two special symmetric networks, and use the outage probabilities of these two symmetric networks as the upper bound and the lower bound of the outage probability of the original asymmetric network, respectively. Specifically, the upper bound and the lower bound of the system outage probability are derived relying on the following lemma.

Lemma. Define $\lambda_{S R_{m}}^{(\min )}$ as $\min \left\{\lambda_{S_{1} R_{m}}, \ldots, \lambda_{S_{N} R_{m}}\right\}$ and $\lambda_{S R_{m}}^{(\max )}$ as $\max \left\{\lambda_{S_{1} R_{m}}, \ldots, \lambda_{S_{N} R_{m}}\right\}$, respectively. Then $\mathrm{F}_{u_{M+1}^{\prime}}(\beta)$ is upper bounded by

$$
\begin{aligned}
& \mathrm{F}_{u_{M+1}^{\prime}}^{(U B)}(\beta) \\
& =\sum_{l=M+1} \sum_{\substack{1 \leq j_{1} \ldots<j_{l} \leq N+M \\
1 \leq j_{l}+\ldots<j_{N+M} \leq N+M \\
j_{1} \neq j_{2} \neq \ldots \neq j_{N+M}}} \prod_{i=1}^{l+M} \mathrm{~F}_{u_{j_{i}}^{(U B)}}(\beta) \\
& \times \prod_{i=l+1}^{N+M}\left(1-\mathrm{F}_{u_{j_{i}}^{(U B)}}(\beta)\right)
\end{aligned}
$$

where

$$
\mathrm{F}_{u_{k}^{(U B)}}(\beta)=\left\{\begin{array}{c}
1-\exp \left(-\lambda_{S_{k} D} \beta\right), 1 \leq k \leq N \\
1-\exp \left(-\lambda_{S R_{k-N}}^{(\max )} \beta-\lambda_{R_{k-N} D} \beta\right) \\
\times K_{1}\left(2 \sqrt{\lambda_{S R_{k-N}}^{(\max )} \lambda_{R_{k-N} D}\left(\beta^{2}+\beta\right)}\right) \\
\times 2 \sqrt{\lambda_{S R_{k-N}(\max )} \lambda_{R_{k-N} D}\left(\beta^{2}+\beta\right)}, \text { else }
\end{array}\right.
$$

and lower bounded by

$$
\begin{aligned}
& \mathrm{F}_{u_{M+1}^{\prime}}^{(L B)}(\beta) \\
& =\sum_{l=M+1} \sum_{\substack{1 \leq j_{1} \ldots<j_{l} \leq N+M \\
1 \leq j_{l+1} \ldots<j_{N+M} \leq N+M \\
j_{1} \neq j_{2} \neq \ldots \neq j_{N+M}}} \prod_{i=1}^{l} \mathrm{~F}_{u_{j_{i}}^{(L B)}}(\beta) \\
& \times \prod_{i=l+1}^{N+M}\left(1-\mathrm{F}_{u_{j_{i}}^{(L B)}}(\beta)\right) \text {, }
\end{aligned}
$$


where

$$
\mathrm{F}_{u_{k}^{(L B)}}(\beta)=\left\{\begin{array}{c}
1-\exp \left(-\lambda_{S_{k} D} \beta\right), 1 \leq k \leq N \\
1-\exp \left(-\lambda_{S R_{k-N}}^{(\min )} \beta-\lambda_{R_{k-N} D} \beta\right) \\
\times K_{1}\left(2 \sqrt{\lambda_{S R_{k-N}}^{(\min )} \lambda_{R_{k-N} D}\left(\beta^{2}+\beta\right)}\right) \\
\times 2 \sqrt{\lambda_{S R_{k-N}}^{(\min )} \lambda_{R_{k-N} D}\left(\beta^{2}+\beta\right)} \text {. else }
\end{array}\right.
$$

Proof: Please refer to Appendix B.

Intuitively, the asymmetric nature of the network would not impact the diversity order performance, thus a diversity order of $M+1$ is supposed to be achieved as in symmetric networks. To verify this point rigorously, with the same procedure used to arrive at (22), the approximated upper bound and lower bound of system outage probability for the asymmetric networks in the high SNR regime are derived as ${ }^{4}$

$$
\begin{aligned}
& \mathrm{F}_{\substack{\left.u_{M+1}^{\prime} \\
u^{\prime} B\right)}}^{(\beta)} \\
& \approx \sum_{i=1}^{M} \sum_{\substack{1 \leq j_{1} \ldots<j_{i} \leq M \\
1 \leq j_{i+1} \ldots<j_{M+1} \leq N}} \prod_{t=1}^{i} \beta\left(\lambda_{S R_{j_{t}}}^{(\max )}+\lambda_{R_{j_{t}} D}\right) \prod_{t=i+1}^{M+1} \beta \lambda_{S_{j_{t}} D} \\
& \propto\left(\frac{N_{0}}{E_{b}}\right)^{M+1}
\end{aligned}
$$

and

$$
\begin{aligned}
& \mathrm{F}_{u_{M+1}^{\prime}}^{(L B)}(\beta) \\
\approx & \sum_{i=1}^{M} \sum_{\substack{1 \leq j_{1} \ldots<j_{i} \leq M \\
1 \leq j_{i+1} \cdots<j_{M+1} \leq N}} \prod_{t=1}^{i} \beta\left(\lambda_{S R_{j_{t}}}^{(\min )}+\lambda_{R_{j_{t}} D}\right) \prod_{t=i+1}^{M+1} \beta \lambda_{S_{j_{t}} D} \\
\propto & \left(\frac{N_{0}}{E_{b}}\right)^{M+1}
\end{aligned}
$$

respectively. From (29) and (31), we conclude that the proposed R3SSP achieves full cooperative diversity order of $M+1$ in asymmetric networks. It should be mentioned that there are $N$ sources and $M$ relays in the entire network, thus a diversity order of $N+M+1$ is achievable when both cooperative diversity and multiuser diversity are obtained [17], [18]. Note that unlike the schemes in [17], [18] where only the "best" source is allowed for transmission, we exploit round-robin scheduling to allocate the opportunities of transmission equally to all the users in R3SSP. Although round-robin scheduling would cause the loss of multiuser diversity, in this paper we focus on the issue of achieving of full cooperative diversity. It is also noted that if R3SSP exploits the greedy scheduler to select the "best" source in each time slot of the broadcast phase, R3SSP obtains both cooperative diversity and multiuser diversity, which results in a total diversity order of $N+M+1$ as [17], [18].

\footnotetext{
${ }^{4}$ These approximations hold under the same limiting condition as that of ${ }^{4}$ Thess.
}

\section{Diversity Multiplexing Tradeoff}

The DMT theory proposed by Zheng and Tse [29] is a fundamental and comprehensive performance metric to characterize both reliability and spectral efficiency of a communication system in high SNR regime. In this subsection, the DMT performance of the proposed scheme is analyzed to verify its superiority. To derive the DMT of the proposed scheme, the relevant definitions are presented below.

In a data-rate adaptive communication system with data rate $\mathcal{R}$ bit/s/Hz, where $\mathcal{R}$ is set to be a function of $\rho$, the multiplexing gain is defined as [29]

$$
r=\lim _{\rho \rightarrow \infty} \frac{\mathcal{R}(\rho)}{\log (\rho)} .
$$

Denote $P_{\text {out }}(\mathcal{R}(\rho))$ as the average system outage probability for the given average $\operatorname{SNR} \rho$ and data rate $\mathcal{R}(\rho)$. The diversity gain is defined as [29]

$$
d=-\lim _{\rho \rightarrow \infty} \frac{\log P_{\text {out }}(\mathcal{R}(\rho))}{\log (\rho)} .
$$

This definition is also written as $P_{\text {out }}(\mathcal{R}(\rho)) \doteq \rho^{-d}$ in the exponential equality notation as used in [29].

With the above definitions, the following theorem is derived.

Theorem. An $N$-source $M$-relay R3SSP with multiplexing gain $r$ achieves the DMT of

$$
d=(M+1)\left(1-\frac{N+M}{N} r\right)^{+},
$$

where $(x)^{+}$represents $\max \{x, 0\}$.

Proof: Please see Appendix C.

It is well known that traditional protocols with optimal relay selection and round-robin relaying achieve the DMT of $(M+1)(1-2 r)^{+}$and $(M+1)(1-(M+1) r)^{+}$, respectively [2], [15]. Clearly, in the network where number of the sources is higher than that of the relays, the proposed protocol provides better DMT performance, i.e. R3SSP achieves higher spectral efficiency than traditional protocols while maintaining the same level of reliability, or it provides larger diversity gain than traditional protocols with the same data rate. As an example, we illustrate the DMT curves for various communication strategies in a five-source three-relay network in Fig. 4. It can be seen that the diversity gain $d$ of R3SSP decreases slower than that of the existing protocols as the multiplexing gain $r$ increases.

\section{ADAPTIVE RELAY ACTIVATION}

In this section, we aim to further improve the DMT performance of the proposed R3SSP in a data-rate adaptive system. To accomplish this goal, the impact of the number of relays on DMT is discussed based on the analytical result, and an ARA scheme is proposed to achieve better DMT.

According to (34), in a network with fixed number of sources, the achievable diversity gain increases in the low multiplexing gain region but decreases in the high multiplexing gain region as the number of relays increases. Take the fivesource three-relay network as an example, it can be calculated 


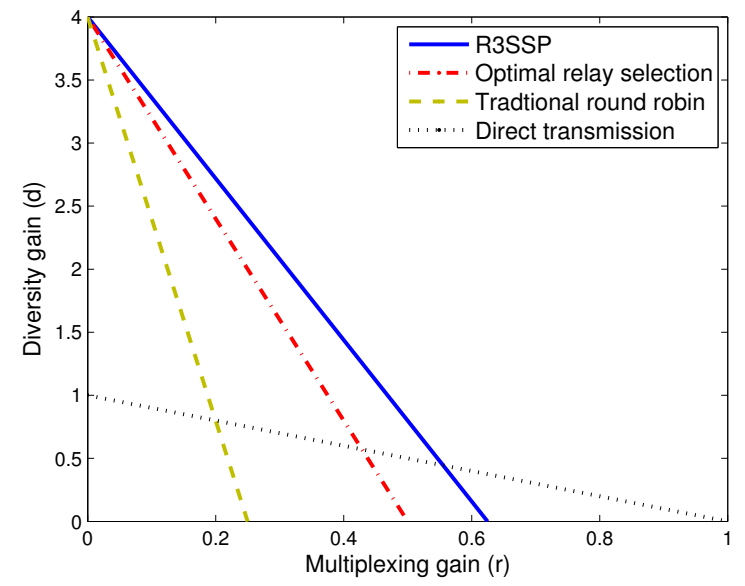

Fig. 4. An example of DMT comparison among the proposed R3SSP, the optimal relay selection scheme and the traditional round-robin scheme. There are five sources and three relays in the network (i.e., $N=5, M=3$ ).

that the three-relay network provides the highest diversity gain in the low multiplexing gain region. As multiplexing gain increases, in the medium multiplexing gain region, the two-relay network and the one-relay network achieve the best diversity gain successively. In the high multiplexing gain region, the network employing only direct transmission harvests the most diversity gain than the other three networks considered.

This result indicates that it might be unwise to activate all the available relays in the entire network. A better way is to select the optimal (in terms of DMT) number of the activated relays with regard to the desired multiplexing gain. Furthermore, turning off some unnecessary relays also leads to a better energy efficiency. Therefore, based on (34), we propose an ARA scheme which dynamically turns on a proper amount of the relays to achieve optimal DMT performance. To be specific, for an $N$-source $M$-relay network with multiplexing gain $r$, the optimal number of the active relay is given as

$$
M^{*}(r)=\arg \max _{m=0, \ldots, M}(m+1)\left(1-\frac{N+m}{N} r\right)^{+} .
$$

It is noted that to achieve the minimum system outage probability, the optimal selection of the $M^{*}(r)$ activated relays is based on (40). However, (34) shows that the DMT performance only depends on the number of the activated relays in a $N$-source network with multiplexing gain $r$, regardless of the fact which particular relays are chosen to serve. Therefore, we can randomly activate $M^{*}(r)$ relays to reduce the computational cost while achieving the optimal DMT.

This sort of ARA strategy can be extended into the traditional relay selection based and round-robin based protocols straightforwardly. For the traditional relay selection based protocols, if $m \geq 1$, the DMT of $(m+1)(1-2 r)^{+}$can be achieved. Otherwise if $m=0$, which indicates only the direct transmission is used, a DMT of $(1-r)^{+}$is obtained. In summary, the relay selection based protocols achieve the DMT of $(m+1)(1-\min \{m+1,2\} \times r)^{+}$for $0 \leq m \leq M$. It can be seen that the optimal number of activated relays is $M$ or 0 in the traditional relay selection based protocols. Therefore, it is always beneficial to use all the relays in the entire network if direct transmission is not optimal, i.e., the optimal number of activated relays is

$$
\begin{aligned}
& M_{(T R S)}^{*}(r) \\
= & \arg \max _{m=0, \ldots, M}(m+1)(1-\min \{m+1,2\} \times r)^{+} \\
= & M \text { or } 0 .
\end{aligned}
$$

In other words, in the traditional relay selection based protocols, it is suggested to switch between activating all the relays and hibernating all the relays. In the traditional round-robin relaying protocols, the optimal number of activated relay is

$$
M_{(T R R)}^{*}(r)=\arg \max _{m=0, \ldots, M}(m+1)(1-(m+1) r)^{+} .
$$

Apparently, in a network where there are more sources than relays, since $\left(1-\frac{N+m}{N} r\right)^{+} \geq(1-\max \{m+1,2\} \times r)^{+} \geq$ $(1-(M+1) r)^{+}$,for all $0 \leq m \leq M$, we have

$$
M^{*}(r) \geq M_{(T R S)}^{*}(r) \geq M_{(T R R)}^{*}(r) .
$$

Therefore, the proposed R3SSP also outperforms its counterparts when ARA strategy is invoked in all the schemes considered.

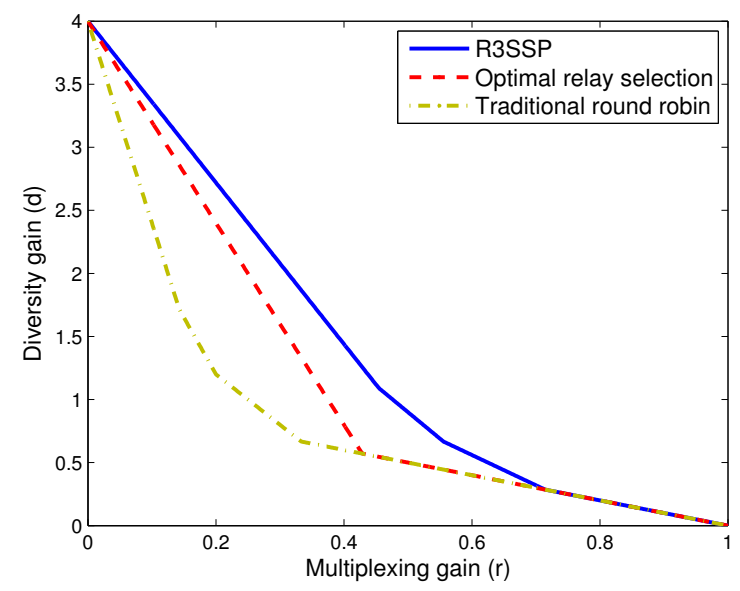

Fig. 5. Comparison of different protocols using ARA in a five-source network.

Consider the five-source three-relay network as an example. Fig. 5 shows the improvement on the DMT performance by employing the ARA strategy. It can be seen from Fig. 5 that the ARA leads to significant improvement on the DMT performance for all the protocols considered, and the proposed R3SSP always achieves better or at least equal performance compared to the existing protocols.

\section{Simulation Results}

In this section, numerical results are presented to demonstrate the performance of the proposed R3SSP in terms of the average system outage probability with different values of the multiplexing gain, and the results corroborate the validity of the proposed R3SSP and consolidate the analytical results presented in the previous sections. The horizontal axis $E_{b} / N_{0}$ 
, defined in Section IV-A, represents the ratio of the average transmit energy per bit to the noise power spectral density in the network. The simulations are performed over Rayleigh block fading channels with AWGNs. We consider two sorts of transmission strategy: the fixed data rate strategy and the datarate adaptive strategy. For the fixed data-rate strategy, $\mathcal{R}$ is set to be a constant and is unrelated to the average SNR of the network. For the data-rate adaptive strategy, $\mathcal{R}$ is a variable which matches the transmission rate with the average SNR of the network.

\section{A. Fixed Data Rate Transmission}

Two scenarios are considered: the symmetric scenario and the asymmetric scenario. In the symmetric network, all the links are assumed to have equal average SNR $\left(\gamma_{S_{n} R_{m}}=\right.$ $\left.\gamma_{R_{m} D}=\gamma_{S_{n} D}, 1 \leq n \leq N, 1 \leq m \leq M\right)$. The asymmetric network is generated in a two-dimensional plane where $D$ is located at the coordinates of $(1,1)$, and the other nodes are uniformly distributed in the first quadrant of the $1 \times 1$ square. The path loss exponent is 2 .

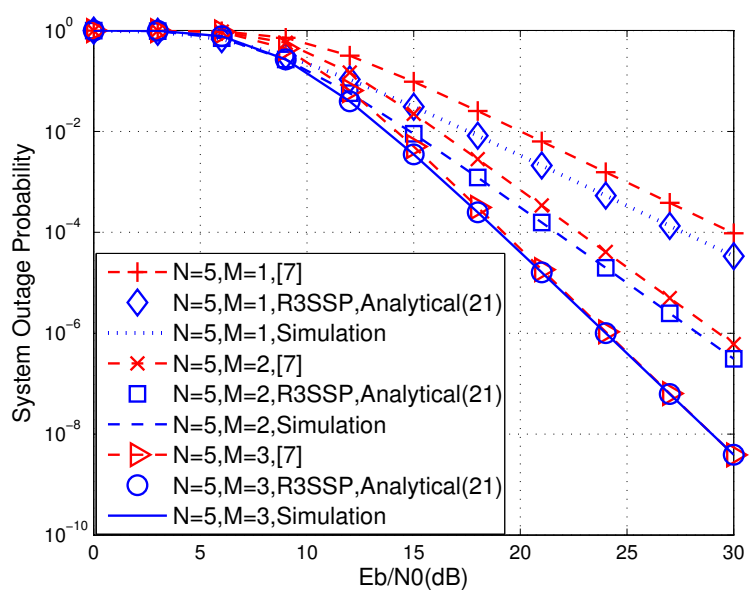

Fig. 6. Outage probability comparisons between the traditional protocol with optimal relay selection [7] and R3SSP under fixed data rate $\mathcal{R}=1 \mathrm{bit} / \mathrm{s} / \mathrm{Hz}$ in the symmetric network. $(N=5)$

From Fig. 6 to Fig. 9 we show the system outage probabilities of the proposed R3SSP and the protocol with optimal relay selection [7] under fixed data rate in the five-source networks. Both the symmetric scenario and the asymmetric scenario are considered. Simulation results of Fig. 6 to Fig. 9 show that R3SSP achieves the same diversity order of $M+1$ as the optimal relay selection protocol does under fixed data rate. Moreover, it is observed from Fig. 6 to Fig. 9 that, when the number of relays is small, in practice R3SSP attains a considerable improvement over the optimal relay selection based protocol [7] in terms of the system outage probability. For example, when the number of relays is $M=1$ or $M=2$, this improvement is up to $2 \sim 3.5 d B$ at system outage probability of $10^{-4}$. Furthermore, theoretic curves of R3SSP are provided. From Fig. 6 and Fig. 7, it can be seen that the analytical results of the system outage probabilities (21) match the simulation results perfectly in the symmetric network. Fig.

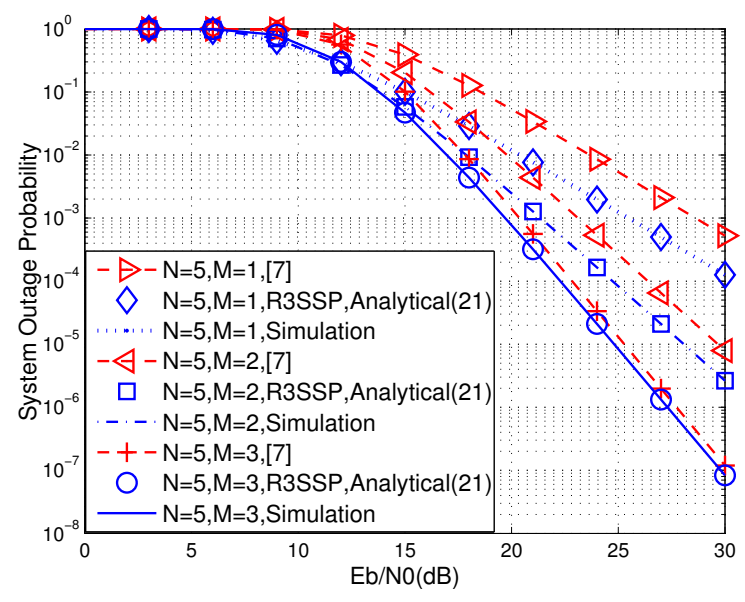

Fig. 7. Outage probability comparisons between the traditional protocol with optimal relay selection [7] and R3SSP under fixed data rate $\mathcal{R}=$ $1.5 \mathrm{bit} / \mathrm{s} / \mathrm{Hz}$ in the symmetric network. $(N=5)$

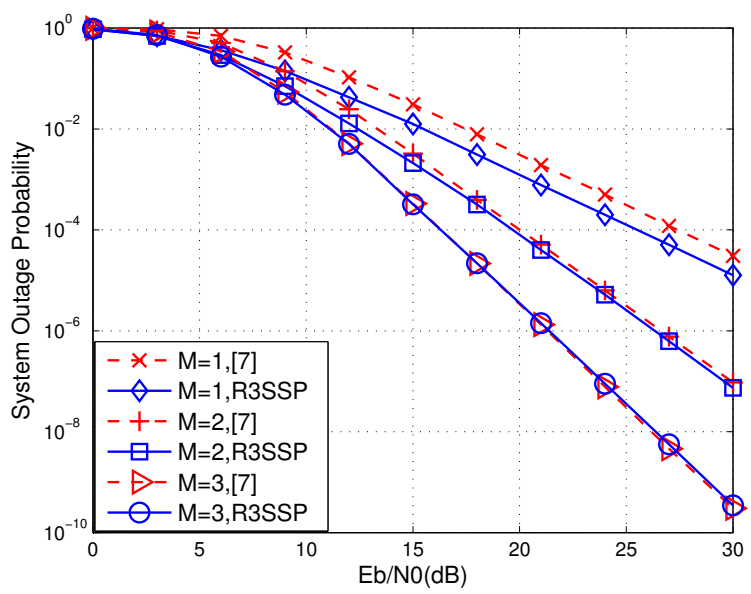

Fig. 8. Outage probability comparisons between the traditional protocol with optimal relay selection [7] and R3SSP under fixed data rate $\mathcal{R}=1 \mathrm{bit} / \mathrm{s} / \mathrm{Hz}$ in the asymmetric network. $(N=5)$

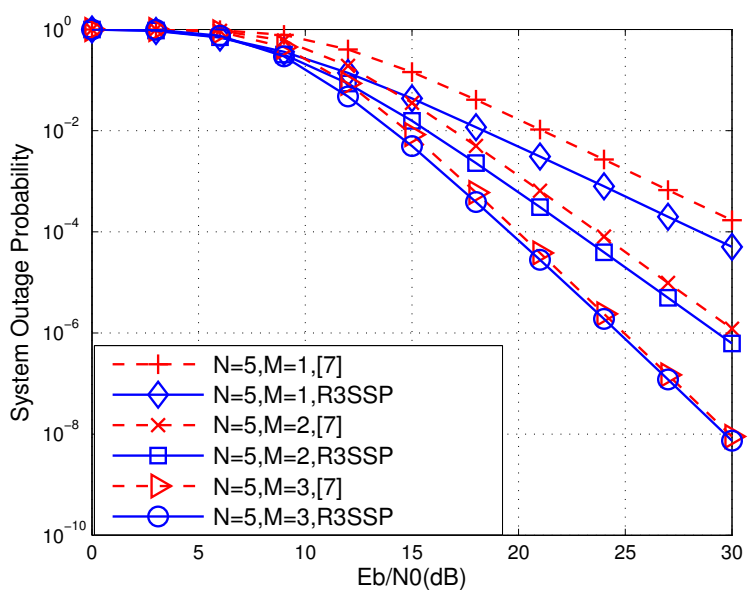

Fig. 9. Outage probability comparisons between the traditional protocol with optimal relay selection [7] and R3SSP under fixed data rate $\mathcal{R}=$ $1.5 \mathrm{bit} / \mathrm{s} / \mathrm{Hz}$ in the asymmetric network. $(N=5)$ 


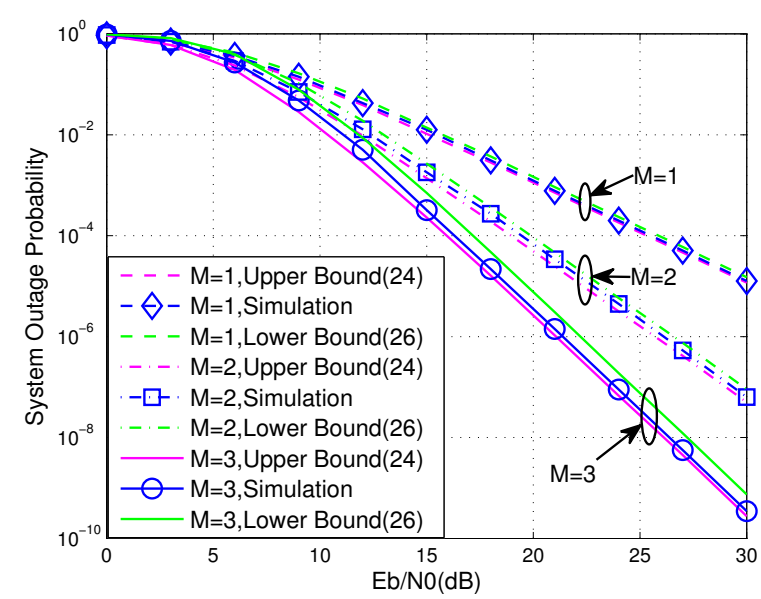

Fig. 10. The upper bound (24) and the lower bound (26) of system outage probability in the asymmetric network under fixed data rate $\mathcal{R}=1 \mathrm{bit} / \mathrm{s} / \mathrm{Hz}$. $(N=5)$

10 illustrates the upper bound (24) and the lower bound (26) of R3SSP in the asymmetric network. It is shown that the simulation curves reside between the upper bound and the lower bound, and that both the upper bound and the lower bound are very tight and indicate a diversity order of $M+1$. To conclude, the analysis in Section IV is validated by the simulations. Therefore, we contend that R3SSP is able to provide full cooperative diversity with round-robin relaying as achieved by [7].

Besides, two facts are observed from Fig. 6 to Fig. 9. First, the performance advantage of R3SSP over the scheme of [7] gradually grows as $\mathcal{R}$ increases. Second, the performance advantage of R3SSP over the scheme of [7] gradually reduces as $M$ increases. The reason is that R3SSP lowers down the required transmission rate of each link by allocating more time resource to direct transmission. To be specific, in order to achieve an end-to-end data rate of $\mathcal{R}$ bit/s/Hz, the actual data rate of each link in R3SSP and the scheme of [7] is $\frac{N+M}{N} \mathcal{R} \mathrm{bit} / \mathrm{s} / \mathrm{Hz}$ and $2 \mathcal{R} \mathrm{bit} / \mathrm{s} / \mathrm{Hz}$, respectively, because $\frac{N}{N+M}$ and $\frac{1}{2}$ of all the time resource available are allocated to transmit "fresh" data in R3SSP and the scheme of [7], respectively. Hence the gap of the required data rate in each link between R3SSP and the scheme of [7] grows as $\mathcal{R}$ increases and reduces as $M$ increases. It implies that R3SSP is capable of supporting higher-rate data transmission than the scheme of [7]. Moreover, it should be noted that as $M$ increases, the complexity of the relay selection in [7] also increases due to the fact that more specific CSI feedback channels are required. By contrast, R3SSP requires no specific CSI feedback and hence can be implemented with lower complexity.

Additionally, in Fig. 11 we provide the system outage probability comparison between R3SSP and the GFNC based scheme of [25] in the context of an asymmetric network composed of two sources, two relays and one destination. We extend R3SSP to DF systems so that both schemes can be fairly compared in a common system. There are four time slots in the GFNC based scheme. In the first and the second

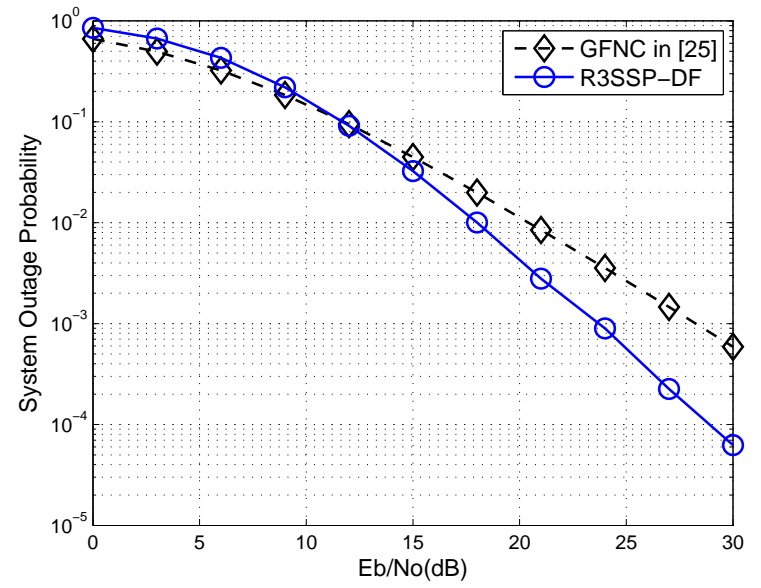

Fig. 11. System outage probability comparison of the GFNC based scheme of [25] and R3SSP under fixed data rate $\mathcal{R}=1 \mathrm{bit} / \mathrm{s} / \mathrm{Hz}$, where SC is used. $(N=M=2)$

time slots, $S_{1}$ and $S_{2}$ broadcast $x_{1}$ and $x_{2}$, respectively, and $R_{1}$ decodes $x_{1}$ and $x_{2}$ as $\hat{x}_{1}$ and $\hat{x}_{2}$, respectively, whereas $R_{2}$ decodes $x_{1}$ and $x_{2}$ as $\tilde{x}_{1}$ and $\tilde{x}_{2}$, respectively. Then in the third and the fourth time slots, $R_{1}$ and $R_{2}$ forward $\hat{x}_{1} \oplus \hat{x}_{2}$ and $\tilde{x}_{2}$, respectively (i.e., we use NC-3 of [25] as the network coding scheme). As shown in Fig. 11, the diversity order of the GFNC based scheme is two, which is lower than the diversity order of three achieved by R3SSP. The reason is that the GFNC based scheme cannot guarantee correct decoding of both $x_{1}$ and $x_{2}$ if $D$ obtains two wrong estimates during the whole transmission. More specifically, if $D$ fails to correctly decode $x_{1}$ from the direct link and $\hat{x}_{1} \oplus \hat{x}_{2}$ from the relay link, $D$ is not able to recover $x_{1}$. Therefore, the GFNC based scheme provides a diversity order of two to $S_{1}$ although it provides a diversity order of three to $S_{2}$. However, the overall diversity order of the entire network depends on the "worst" source, hence the diversity order of the network is two. In contrast, R3SSP always improves the "worst" source's performance, and provides a diversity order of three for all sources. Therefore, we contend that R3SSP achieves a more efficient relay sharing among all sources.

\section{B. Adaptive Data Rate Transmission}

Simulations are also performed under the adaptive data rate strategy to show the DMT performance of R3SSP and the scheme of [7]. In the adaptive data rate strategy, the desired data rate is determined by the average SNR of the network and the multiplexing gain as $\mathcal{R}=r \log \left(1+\frac{E_{b}}{N_{0}}\right)+c$, where $c$ is a constant and set to be zero in the following simulations. ${ }^{5}$ As analyzed and verified previously, both the symmetric networks and the asymmetric network achieve the same diversity order. Therefore, we consider the five-source three-relay asymmetric network which is generated in the same fashion as the asymmetric network of the above subsection.

\footnotetext{
${ }^{5}$ Obviously, the fixed data rate strategy is a special case of the adaptive data rate strategy when $r=0$ and $c$ is a positive constant.
} 


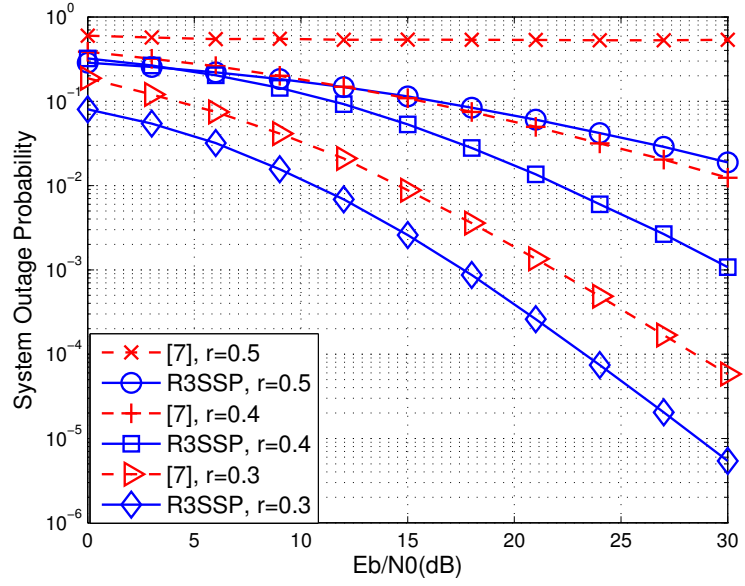

Fig. 12. Outage probability comparisons between the traditional protocol with optimal relay selection [7] and R3SSP in the asymmetric network under different multiplexing gains. $(N=5, M=3)$

Fig. 12 illustrates the system outage probabilities of R3SSP and its counterpart in [7] with a range of different multiplexing gains. The result agrees with the DMT analysis and shows that R3SSP achieves higher diversity gain than the scheme of [7] when $r>0$. It is also shown that the performance advantage of R3SSP over its counterpart in [7] becomes more significant as $r$ increases. This is because the diversity gain reduces faster in the relay selection based protocols than in R3SSP when $r$ becomes larger. For example, the diversity gain of R3SSP reduces from 2.08 to 1.44 , whereas the diversity gain of the scheme of [7] reduces from 1.6 to 0.8 , when $r$ grows from 0.3 to 0.4 .

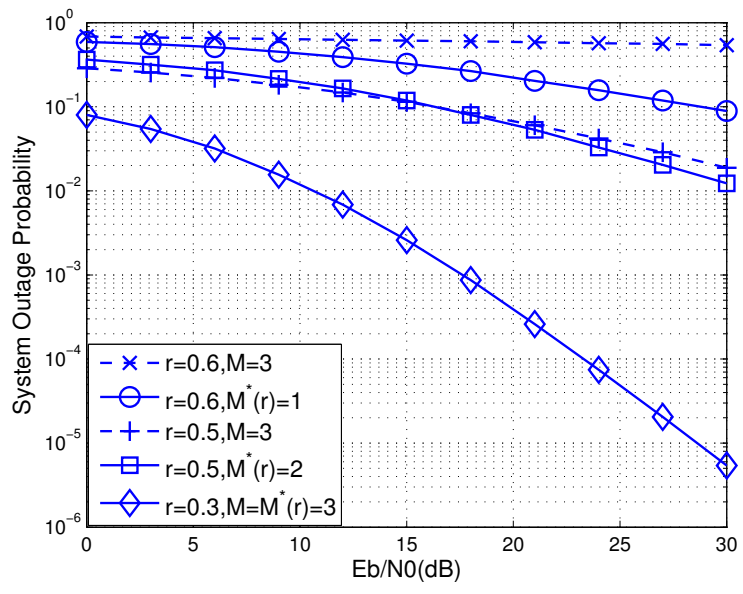

Fig. 13. The performance improvement of ARA in R3SSP. The dashed and the solid lines are the system outage curves of R3SSP with and without ARA, respectively. $(N=5, M=3)$

Fig. 13 shows the improvement of the system outage performance caused by the ARA in R3SSP. According to (35), we randomly select $M^{*}(r)$ relays to serve for improving the DMT performance. As observed from Fig. 13, it is not always optimal to use all the relays to assist transmission in R3SSP. The ARA strategy achieves better diversity gain and hence significantly reduces the system outage probability by hibernating unnecessary relays according to the desired multiplexing gain and the average SNR of the network, which also improves the energy efficiency of the network .

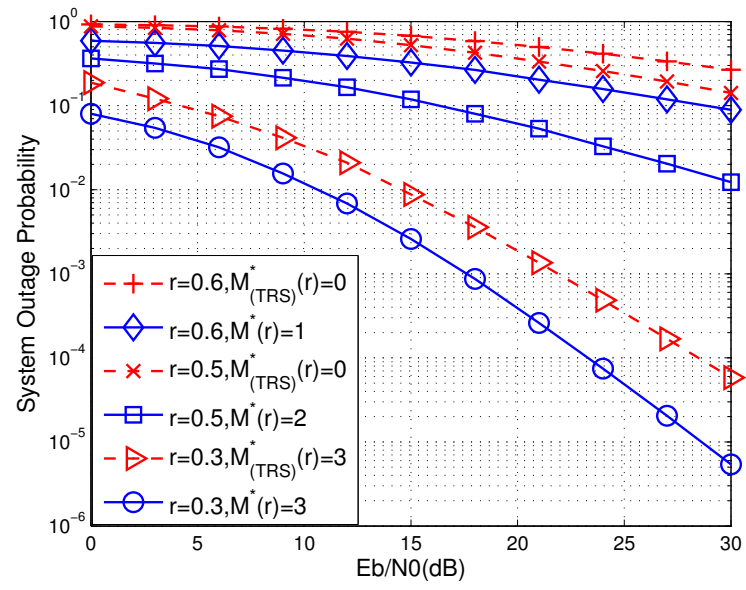

Fig. 14. Comparisons between R3SSP and the scheme of [7], both with ARA. $(N=5, M=3)$

Comparisons between R3SSP and the optimal relay selection protocol of [7] are illustrated in Fig. 14, where both protocols use the ARA strategy. Unsurprisingly, with the ARA strategy, R3SSP still performs better. As we analyzed in Section IV, the reason accounting for this superiority is twofold: i) R3SSP achieves better DMT than the scheme of [7] when both protocols turn on the same amount of relays; ii) R3SSP provides more flexible usage of the relays than the optimal relay selection protocol of [7] which only makes a binary decision: to use all the relays or to use none of the relays.

From Fig. 12 to Fig. 14, it can be summarized that R3SSP significantly improves the DMT performance and constitutes a more efficient resource-use strategy than the traditional relay selection based protocols. As a result, R3SSP is well-suited for the uplink of future wireless communication systems which are expected to serve an enormous amount of users. Furthermore, the proposed ARA strategy is proved to be effective to further enhance the DMT performance of R3SSP whilst improving the energy efficiency of the network.

\section{CONCLUSIONS}

A novel relay protocol R3SSP is proposed in this paper, which works in a round-robin manner and dispenses with relay selection. Compared with the traditional relay protocols using relay selection to achieve full cooperative diversity, the proposed R3SSP requires no CSI feedback, hence it is featured with a lower implementation complexity in practice. Moreover, the outage probability and DMT performance are investigated. Theoretical analysis indicates that R3SSP achieves better DMT than the traditional protocols in the uplink of a centralized wireless network such as LTE and WiMAX. It means that R3SSP significantly improves the system throughput whilst maintaining the same level of reliability, or R3SSP enjoys a 
much more reliable transmission with the same data rate. An ARA strategy is also proposed to further improve the DMT performance of R3SSP. Simulation results corroborate the effectiveness of R3SSP and show that the proposed protocol outperforms the existing protocols in terms of the DMT performance and the implementation complexity.

\section{APPENDIX A \\ THE CALUCATION OF THE OUTAGE PROBABILITY OF THE ASYMMETRIC NETWORK}

As mentioned in Section IV-B, for a given source-selection ordering, $u_{N+m}$ obeys a distribution having deterministic parameters. Therefore, (14) can be written as the sum of a series of probabilities that a given source-selection ordering and system outage occur simultaneously, i.e.,

$$
\begin{aligned}
P_{\text {out }}(\mathcal{R}) & =\operatorname{Pr}\left(u_{M+1}^{\prime} \leq \beta\right) \\
& =\sum_{\Psi} \operatorname{Pr}\left(u_{M+1}^{\prime} \leq \beta ; \psi_{1}, \ldots, \psi_{N+M}\right)
\end{aligned}
$$

where $\operatorname{Pr}\left(u_{M+1}^{\prime} \leq \beta ; \psi_{1}, \ldots, \psi_{N+M}\right)$ denotes the probability that both the system outage event and the source-selection ordering $\left(\psi_{1}, \ldots, \psi_{N+M}\right)$ take place, and is formulated as

$$
\begin{aligned}
& \operatorname{Pr}\left(u_{M+1}^{\prime} \leq \beta ; \psi_{1}, \ldots, \psi_{N+M}\right) \\
= & \int_{0}^{\beta} \mathrm{f}_{u_{\psi_{M+1}}}\left(t_{M+1} \mid \psi_{1}, \ldots, \psi_{N+M}\right) \\
& \times \operatorname{Pr}\left(u_{\psi_{1}}, \ldots, u_{\psi_{l}} \leq \beta<u_{\psi_{l+1}}, \ldots, u_{\psi_{N+M}}\right) d t_{M+1} \\
= & \int_{t_{N+M-1}}^{\infty} \ldots \int_{t_{M+1}}^{\infty} \int_{0}^{\beta} \int_{0}^{t_{M+1}} \ldots \int_{0}^{t_{2}} \\
& \prod_{k=1}^{N+M} \mathrm{f}_{u_{\psi_{k}}}\left(t_{k} \mid \psi_{1}, \ldots, \psi_{N+M}\right) d t_{1} \ldots d t_{N+M}
\end{aligned}
$$

where $\mathrm{f}_{u_{\psi_{k}}}\left(. \mid \psi_{1}, \ldots, \psi_{N+M}\right)$ is the probability density function (PDF) of $u_{\psi_{k}}$ under the condition that the source-selection ordering is $\left(\psi_{1}, \ldots, \psi_{N+M}\right)$.

To calculate (40), we need to obtain the expression of $\mathrm{f}_{u_{\psi_{k}}}\left(. \mid \psi_{1}, \ldots, \psi_{N+M}\right)$. As pointed out in Section IV$\mathrm{A}$, there exists and only exists one source-selection result $\left(\theta_{1}, \ldots, \theta_{M}\right)$ corresponding to any given source-selection ordering $\left(\psi_{1}, \ldots, \psi_{N+M}\right) .\left(\theta_{1}, \ldots, \theta_{M}\right)$ can be found out using a specific procedure to be detailed below. The procedure determines which source's information is the most unreliable and hence is required to be relayed in each relay time slot of R3SSP according to the given source-selection ordering $\left(\psi_{1}, \ldots, \psi_{N+M}\right)$. The detail of the procedure is summarized in pseudocode form as shown in Table I. It should be noted that this procedure is used to obtain the exact expression of the system outage probability of R3SSP, rather than to carry out R3SSP.

According to the source-selection result $\left(\theta_{1}, \ldots, \theta_{M}\right)$, the CDF of $u_{k}$ under a given source-selection ordering $\left(\psi_{1}, \ldots, \psi_{N+M}\right)$ is presented as follows. When $1 \leq \psi_{k} \leq N$, $u_{\psi_{k}}$ is the SNR of the direct link $S_{\psi_{k}} \rightarrow D$ and its CDF is given as

$$
\mathrm{F}_{u_{\psi_{k}}}\left(x \mid \psi_{1}, \ldots, \psi_{N+M}\right)=1-\exp \left(-\lambda_{S_{\psi_{k}} D} x\right) .
$$

TABLE I

\begin{tabular}{l} 
The procedure of calculating $\left(\theta_{1}, \ldots, \theta_{M}\right)$ \\
\hline 1) Define $m$ as the current relay time slot. As shown previously, in \\
each relay time slot, the quality record of each source (i.e., $\xi_{n}^{(m)}$, \\
$n=1, \ldots, N$,) can be represented by an element of U. Define $w_{n}$ as \\
the index that indicates which element of $\mathrm{U}$ denoting the quality record \\
of source $n$ in the current relay time slot, i.e., $\xi_{n}^{(m)}=u_{w_{n}}$. Initialize \\
$w_{n}=n$, and $m=1$. \\
2) Since the source-selection ordering is known, we can figure out which \\
source's quality record is the "worst" in the current relay time slot. \\
Compute $\theta_{m}=\arg { }_{k=1, \ldots, N+M} \prod_{n=1}^{N}\left(\psi_{k}-w_{n}\right) \mid$. \\
3) Update the index $w_{n}$ after current relay's transmission, and prepare for \\
the next relay time slot. Compare $u_{N+m}$ with $u_{w_{\theta}}$. If $u_{N+m}>u_{w_{\theta}}$, \\
then $w_{\theta_{m}}=N+m$; otherwise, $w_{\theta_{m}}=w_{\theta_{m}}$. Update $m=m+1$. \\
4) Repeat step 2 and 3 until $m=M$.
\end{tabular}

When $N+1 \leq \psi_{k} \leq N+M, u_{\psi_{k}}$ is the SNR of the two-hop link $S_{\theta_{\psi_{k-N}}} \rightarrow R_{k-N} \rightarrow D$ and its CDF is [17]

$$
\begin{aligned}
& \mathrm{F}_{u_{\psi_{k}}}\left(x \mid \psi_{1}, \ldots, \psi_{N+M}\right) \\
& =1-\exp \left(-\lambda_{S_{\psi_{\psi_{k-N}}} R_{k-N}} x-\lambda_{R_{k-N} D} x\right) \\
& \quad \times 2 \sqrt{\lambda_{S_{\theta_{\psi_{k-N}}} R_{k-N}} \lambda_{R_{k-N} D} x(x+1)} \\
& \quad \times K_{1}\left(2 \sqrt{\lambda_{S_{\theta_{\psi_{k-N}}} R_{k-N}} \lambda_{R_{k-N} D} x(x+1)}\right) .
\end{aligned}
$$

Therefore, $\mathrm{f}_{\psi_{k}}\left(x \mid \psi_{1}, \ldots, \psi_{N+M}\right)$ can be written as

$$
\mathrm{f}_{u_{\psi_{k}}}\left(x \mid \psi_{1}, \ldots, \psi_{N+M}\right)=\frac{d \mathrm{~F}_{u_{\psi_{k}}}\left(x \mid \psi_{1}, \ldots, \psi_{N+M}\right)}{d x} .
$$

By substituting (41) and (42) into (43), the closed form expression of $\mathrm{f}_{\psi_{k}}\left(x \mid \psi_{1}, \ldots, \psi_{N+M}\right)$ is given as (44) and (45) (Please refer to Appendix B). Then by substituting (44), (45) into (40), the exact system outage probability can be calculated numerically in principle.

\section{APPENDIX B}

THE EXPRESSION OF $\mathrm{f}_{u_{\psi_{k}}}\left(x \mid \psi_{1}, \ldots, \psi_{N+M}\right)$ If $1 \leq \psi_{k} \leq N$,

$$
\begin{aligned}
& \mathrm{f}_{u_{\psi_{k}}}\left(x \mid \psi_{1}, \ldots, \psi_{N+M}\right)=\lambda_{S_{\psi_{k}} D} \times \exp \left(-\lambda_{S_{\psi_{k}} D} x\right) \text {. } \\
& \text { If } N+1 \leq \psi_{k} \leq N+M \text {, } \\
& \mathrm{f}_{u_{\psi_{k}}}\left(x \mid \psi_{1}, \ldots, \psi_{N+M}\right) \\
& =\exp \left(-\lambda_{S_{\psi_{\psi_{k-N}}} R_{k-N}} x-\lambda_{R_{k-N} D} x\right) \times \\
& \left\{K_{1}\left(2 \sqrt{\lambda_{S_{\psi_{k-N}} R_{k-N}} \lambda_{R_{k-N} D} x(x+1)}\right) \times\right. \\
& \left(2 \sqrt{\lambda_{S_{\theta_{\psi_{k-N}}} R_{k-N} \lambda_{R_{k-N} D} x(x+1)}}\right. \\
& \left.-\lambda_{S_{\theta_{\psi_{k-N}}} R_{k-N}} \lambda_{R_{k-N} D}(2 x+1)\right)+ \\
& \left(K_{0}\left(2 \sqrt{\lambda_{S_{\psi_{k-N}} R_{k-N}} \lambda_{R_{k-N} D} x(x+1)}\right)+\right. \\
& \left.K_{2}\left(2 \sqrt{\lambda_{S_{\psi_{\psi_{k-N}}} R_{k-N}} \lambda_{R_{k-N} D} x(x+1)}\right)\right) \\
& \left.\times \lambda_{S_{\theta_{\psi_{k-N}}} R_{k-N}} \lambda_{R_{k-N} D}(2 x+1)\right\},
\end{aligned}
$$


This article has been accepted for publication in a future issue of this journal, but has not been fully edited. Content may change prior to final publication.

where $K_{q}($.$) is the q$ th order modified Bessel function of the second kind, $q=0,1,2$.

\section{APPENDIX C \\ PROOF OF THE LEMMA}

As described in Section III-B, the closed form expression of the system outage probability in the asymmetric networks is rather complicated since the distribution of $u_{N+m}$ is related to the source-selection ordering. $u_{N+m}$ depends on the quality (i.e., average SNR) of both the $S_{\theta_{m}} \rightarrow R_{m}$ and $R_{m} \rightarrow D$ links. Different source-selection orderings result in different quality of the source-to-relay link, thus yield a different distribution of $u_{N+m}$. To derive the upper bound and the lower bound of the system outage probability for a given asymmetric network, we consider two special $(N+M+1)$ node symmetric networks. In the first symmetric network, we assume that the qualities of the source-to-relay links are lower than or equal to those of the corresponding links in the original asymmetric network, and that the qualities of the source-to-destination and the relay-to-destination links are the same as those of the corresponding links in the original asymmetric network. Intuitively, since the quality of each link in the first symmetric network is not better than the corresponding link in the original asymmetric network, it suffers a higher system outage probability. Thus the upper bound is obtained by calculating the system outage probability of this special symmetric network using (21). Similarly, we can generate the second symmetric network where the source-torelay links have higher or identical qualities compared to the corresponding links in the original asymmetric network, and get the lower bound.

For any given network with fading coefficients $\lambda_{S_{n} D}$, $\lambda_{S_{n} R_{m}}$ and $\lambda_{R_{m} D}(1 \leq n \leq N, 1 \leq m \leq M)$, the SNRs of the received signals at $D$ during the whole transmission phase are written as

$$
u_{k}= \begin{cases}\xi_{S_{k} D}, & 1 \leq k \leq N \\ \frac{\xi_{S_{\theta_{k-N}} R_{k-N}} \xi_{R_{k-N} D}}{\xi_{S_{\theta_{k-N}} R_{k-N}+\xi_{R_{k-N} D}+1}}, & N<k \leq N+M\end{cases}
$$

where $\xi_{S_{k} D}, \xi_{S_{n} R_{m}}$ and $\xi_{R_{m} D}$ defined in (3) and (8) obey the exponential distributions with parameters $\lambda_{R_{k} D}, \lambda_{S_{n} R_{m}}$ and $\lambda_{R_{m} D}$, respectively.

Denote $\max \left\{\lambda_{S_{1} R_{m}}, \ldots, \lambda_{S_{N} R_{m}}\right\}$ as $\lambda_{S R_{m}}^{\max }$. Let us construct the first symmetric network where the SNRs of the received signals at $D$ during the whole transmission phase are given as

$$
u_{k}^{(U B)}= \begin{cases}\xi_{S_{k} D}, & 1 \leq k \leq N \\ \frac{\xi_{S R_{k-N}}^{(U B)} \xi_{R_{k-N} D}}{\xi_{S R_{k-N}}^{(U B)}+\xi_{R_{k-N} D}+1}, & N<k \leq N+M\end{cases}
$$

where $\xi_{S R_{k-N}}^{(U B)}=\min \left\{\xi_{S_{\theta_{k-N}} R_{k-N}}, \bar{\xi}_{S_{\theta_{k-N}} R_{k-N}}\right\} \cdot \xi_{S_{k} D}$, $\xi_{S_{n} R_{m}}, \bar{\xi}_{S_{n} R_{m}}$ and $\xi_{R_{m} D}$ obey the exponential distributions with parameters $\lambda_{S_{k} D}, \lambda_{S_{n} R_{m}}, \lambda_{S R_{m}}^{(\max )}-\lambda_{S_{n} R_{m}}$ and $\lambda_{R_{m} D}$, respectively (here we define that when $\lambda_{S R_{m}}^{(\max )}-\lambda_{S_{n} D_{m}}=$ $\left.0, \bar{\xi}_{S_{n} R_{m}}=+\infty\right)$. Obviously, $\xi_{S R_{m}}^{(U B)}$ obeys the exponential distribution with parameter $\lambda_{S R_{m}}^{(\max )}$. It can be seen that $u_{k} \geq u_{k}^{(U B)}$. Let $u_{k}^{(U B)}$ represent the $k$ th maximum value of $\left(u_{1}^{(U B)}, \ldots, u_{N+M}^{(U B)}\right)$, then we have $u_{k}^{\prime} \geq u_{k}^{\prime(U B)}$. Readily, we can prove that $\mathrm{F}_{u_{M+1}^{\prime}}(x) \leq \mathrm{F}_{u_{M+1}^{\prime(U B)}}(x)$, where $\mathrm{F}_{u_{M+1}^{\prime(U B)}}($.$) is$ the CDF of $u_{k}^{\prime(U B)}$. From (21), $\mathrm{F}_{u_{M+1}^{\prime(U B)}}(x)$ is formulated as

$$
\begin{aligned}
& \mathrm{F}_{\substack{u_{M+1}^{\prime(U B)} \\
l=M+1}}(x) \\
= & \sum_{\substack{1 j_{1} \ldots<j_{1} \leq N+M \\
1 \\
j_{l+1} \ldots<j_{N}+M \leq N+M \\
j_{1} \neq j_{2} \neq \ldots \neq j_{N+M}}}^{N+M} \prod_{i=1}^{l} \mathrm{~F}_{u_{j_{i}}^{(U B)}}(x) \\
& \times \prod_{i=l+1}^{N+M}\left(1-\mathrm{F}_{u_{j_{i}}^{(U B)}}(x)\right),
\end{aligned}
$$

where

$$
\mathrm{F}_{u_{k}^{(U B)}}(x)=\left\{\begin{array}{c}
1-\exp \left(-\lambda_{S_{k} D} x\right), 1 \leq k \leq N \\
1-\exp \left(-\lambda_{S R_{k-N}}^{(\max )} x-\lambda_{R_{k-N} D} x\right) \\
\times K_{1}\left(2 \sqrt{\lambda_{S R_{k-N}}^{(\max )} \lambda_{R_{k-N} D}\left(x^{2}+x\right)}\right) \\
\times 2 \sqrt{\lambda_{S R_{k-N}}^{(\max )} \lambda_{R_{k-N} D}\left(x^{2}+x\right)}, \text { else }
\end{array}\right.
$$

As a result, we get the upper bound of $\mathrm{F}_{u_{M+1}^{\prime}}(\beta)$ as (24).

Similarly, denote $\min \left\{\lambda_{S_{1} R_{m}}, \ldots, \lambda_{S_{N} R_{m}}\right\}$ as $\lambda_{S R_{m}}^{(\min )}$. The $\mathrm{RV} \xi_{S_{\theta_{k-N}} R_{k-N}}$ can be derived as the minimum value of two exponential distributed RVs $\xi_{S R_{k-N}}^{(L B)}$ and $\underline{\xi}_{S_{\theta_{k-N}} R_{k-N}}$, where $\xi_{S R_{k-N}}^{(L B)}$ and $\underline{\xi}_{S_{\theta_{k-N}} R_{k-N}}$ obey the exponential distributions with parameters $\lambda_{S R_{m}}^{(\min )}$ and $\lambda_{S_{\theta_{k-N}} R_{k-N}}-\lambda_{S R_{m}}^{(\min )}$ (here we also define that when $\lambda_{S_{\theta_{k-N}} R_{k-N}}-\lambda_{S R_{m}}^{(\min )}=0$, $\left.\underline{\xi}_{S_{\theta_{k-N}} R_{k-N}}=+\infty\right)$, respectively. With the same procedure for deriving the upper bound, the lower bound of $\mathrm{F}_{u_{M+1}^{\prime}}(\beta)$ is obtained as (26).

\section{APPENDIX D}

PROOF OF THE THEOREM

From (33), the diversity gain is calculated as

$$
\begin{aligned}
d & =-\lim _{\rho \rightarrow \infty} \frac{\log P_{\text {out }}(\mathcal{R}(\rho))}{\log \rho} \\
& =-\lim _{\rho \rightarrow \infty} \frac{\log \mathrm{F}_{u_{M+1}^{\prime}}(\beta)}{\log \rho},
\end{aligned}
$$

where

$$
\beta=\rho^{\frac{N}{N+M}} r-1 .
$$

According to (28),

$$
d \geq-\lim _{\rho \rightarrow \infty} \frac{\log \mathrm{F}_{u_{M+1}^{\prime}(U)}^{(U)}(\beta)}{\log \rho}
$$

Note that $\mathrm{F}_{u_{M+1}^{\prime}}^{(U B)}(\beta) \leq 1$, thus we have

$$
-\lim _{\rho \rightarrow \infty} \frac{\log \mathrm{F}_{u_{M+1}^{\prime}}^{(U B)}(\beta)}{\log \rho} \geq 0 .
$$


Similar to (23), (28) holds when $\lim _{\rho \rightarrow \infty} \frac{N \beta}{\rho(N+M)}=0$. Since $\lim _{\rho \rightarrow \infty} \frac{N \beta}{\rho(N+M)}=\lim _{\rho \rightarrow \infty} \frac{N}{N+M} \rho^{\left(\frac{N+M}{N} r-1\right)}$, it can be seen that (28) holds in the case of $r<\frac{N}{N+M}$. Substituting (13) and (28) into the right-hand side of (49), we have

$$
\begin{aligned}
& -\lim _{\rho \rightarrow \infty} \frac{\log \mathrm{F}_{u_{M+1}^{(U B)}(\beta)}^{\prime}}{\log \rho} \\
& =-\lim _{\rho \rightarrow \infty} \frac{\log \left(\rho^{-(M+1)}\left(\frac{N \beta}{N+M}\right)^{M+1}\right)}{\log \rho} \\
& =-\lim _{\rho \rightarrow \infty} \frac{\log \left(\rho^{-(M+1)}\left(\rho^{\frac{N+M}{N} r}\right)^{M+1}\right)}{\log \rho} \\
& =-\lim _{\rho \rightarrow \infty} \frac{\log \left(\rho^{-(M+1)\left(1-\frac{N+M}{N} r\right)}\right)}{\log \rho} \\
& =(M+1)\left(1-\frac{N+M}{N} r\right) \text {, }
\end{aligned}
$$

if $r<\frac{N}{N+M}$. According to (49), (50) and (51), we obtain

$$
d \geq-\lim _{\rho \rightarrow \infty} \frac{\log \mathrm{F}_{u_{M+1}^{\prime}(U)}^{(\beta)}(M)}{\log \rho}=(M+1)\left(1-\frac{N+M}{N} r\right)^{+}
$$

Similarly, we can get

$$
d \leq-\lim _{\rho \rightarrow \infty} \frac{\log \mathrm{F}_{u_{M+1}^{\prime}(\beta)}^{(\beta)}}{\log \rho}=(M+1)\left(1-\frac{N+M}{N} r\right)^{+}
$$

As a result, the DMT of R3SSP is $d=$ $(M+1)\left(1-\frac{N+M}{N} r\right)^{+}$.

\section{REFERENCES}

[1] J. Proakis and M. Salehi, Digital communications, Fifth Edition, McGraw-Hill, New York, 2007

[2] J.N. Laneman, D. Tse, and G.W. Wornell, "Cooperative diversity in wireless networks: efficient protocols and outage behavior," IEEE Transactions on Information Theory, vol. 50, no. 12, pp. 3062-3080, Dec. 2004

[3] M. Peng, H. Liu, W. Wang, and H. Chen, "Cooperative network coding with MIMO transmission in wireless decode-and-forward relay networks," IEEE Transactions on Vehicular Technology, vol. 59, no. 7, pp. 3577-3588, Sept. 2010

[4] Y. Jing and H. Jafarkhani, "Single and multiple relay selection schemes and their achievable diversity orders," IEEE Transactions on Wireless Communications, vol. 8, no. 3, pp. 1414-1423, Mar. 2009

[5] V. Sreng, H. Yanikomeroglu, and D.D. Falconer, "Relayer selection strategies in cellular networks with peer-to-peer relaying," in Proc. IEEE Conf. on Veh. Technol. (VTC) 2003 Fall, Orlando, Florida, USA, Oct. 2003, pp. 1949-1953

[6] Y. Zhao, R. Adve, and T.J. Lim, "Symbol error rate of selection amplifyand-forward relay systems," IEEE Communications Letters, vol. 10, no. 11 , pp. $757-759$, Nov. 2006

[7] A. Bletsas, H. Shin, and M.Z. Win, "Cooperative communications with outage-optimal opportunistic relaying," IEEE Transactions on Wireless Communications, vol. 6, no. 9, pp. 3450-3460, Sept. 2007

[8] A. Bletsas, A. Lippnian, and D. P. Reed, "A simple distributed method for relay selection in cooperative diversity wireless networks based on reciprocity and channel measurements," in Proc. IEEE Conf. on Veh. Technol. (VTC) 2005 Spring, Stockholm, Sweden, May 2005, pp. 1484 1488
[9] R. Madan, N. Mehta, A. Molisch, and J. Zhang, "Energy-efficient cooperative relaying over fading channels with simple relay selection," IEEE Transactions on Wireless Communications, vol. 7, no. 8, pp. 30133025, Aug. 2008

[10] L. Li, X. Zhou, H. Xu, G. Y. Li, and A. Soong, "Simplified relay selection and power allocation in cooperative cognitive radio systems," IEEE Transactions on Wireless Communications, vol. 10, no. 1, pp. 3336, Jan. 2011

[11] D.S. Michalopoulos, N.D. Chatzidiamantis, R. Schober, and GK. Karagiannidis, "Relay selection with outdated channel estimates in Nakagami-m fading," in Proc. IEEE International Conference on Communications (ICC) 2011, Kyoto, Japan, pp. 1-6, Jun. 2011

[12] C. Esli, and A. Wittneben, "A hierarchical AF protocol for distributed orthogonalization in multiuser relay networks," IEEE Transactions on Vehicular Technology, vol. 59, no. 8, pp. 3902-3916, Oct. 2010

[13] S. Yang and R. Koetter, "Network coding over a noisy relay: a belief propagation approach," in Proc. IEEE Int. Symp. Inf. Theory (ISIT) 2007, Nice, France, pp. 801-804, Jun. 2007

[14] C. Hausl, F. Schreckenbach, I. Oikonomidis, and G. Bauch, "Iterative network and channel decoding on a tanner graph," in Proc. Allerton Conf. on Commun., Control and Computing 2005, Monticello, IL, USA, Sept. 2005, pp. 2093-2102

[15] C. Peng, Q. Zhang, M. Zhao, and Y. Yao, "On the performance analysis of network-coded cooperation in wireless networks," in Proc. IEEE Int. Conf. on Computer Commun. (INFOCOM) 2007, Anchorage, Alaska, USA, May 2007, pp. 1460-1468

[16] Y. Chen, S. Kishore, and J. Li, "Wireless diversity through network coding," in Proc. IEEE Wireless Commun. and Networking Conf. (WCNC) 2006, Las Vegas, NV, USA, Apr. 2006, pp. 1681-1686

[17] L. Sun, T. Zhang, L. Lu, and H. Niu, "On the combination of cooperative diversity and multiuser diversity in multi-source multi-relay wireless networks," IEEE Signal Processing Letters, vol. 17, no. 6, pp. 535-538, Jun. 2010

[18] H. Ding, J. Ge, D. Benevides da Costa, and Z. Jiang, "A new efficient low-complexity scheme for multi-source multi-relay cooperative networks," IEEE Transactions on Vehicular Technology, vol. 60, no. 2, pp. 716-722, Feb. 2011.

[19] R. Ahlswede, N. Cai, S.Y.R. Li, and R.W. Yeung, "Network information flow," IEEE Transactions on Information Theory, vol. 46, no. 4, pp. 1204-1216, Jul. 2000

[20] D.R. Pauluzzi and N.C. Beaulieu, "A comparison of SNR estimation techniques for the AWGN channel," IEEE Transactions on Communications, vol. 48, no. 10, pp. 1681-1691, Oct. 2000

[21] A. Wiesel, J. Goldberg, and H. Messer-Yaron, "SNR estimation in time-varying fading channels," IEEE Transactions on Communications, vol. 54, no. 5, pp. 841-848, May 2006

[22] “3GPP-TSG-RSN physical layer general description(release 10)," $3 G P P$ Std. TS 36.211, Mar. 2010

[23] "IEEE draft amendment standard for local and metropolitan area networks-part 16: Air interface for broadband wireless access systemsadvanced air interface," IEEE Std $802.16 \mathrm{~m} / \mathrm{D} 10$, Nov. 2010

[24] A. Nasri, R. Schober, and M. Uysal, "Error rate performance of network-coded cooperative diversity systems", in Proc. IEEE Global Communications Conference (GLOBECOM) 2010, Miami, Florida, USA, pp. 1-6, Dec. 2010

[25] M. Di Renzo, M. Iezzi, and F. Graziosi, "Diversity and coding gain of multi-source multi-relay cooperative wireless networks with binary network coding", http://arxiv.org/pdf/1109.4599v1.pdf

[26] T. Islam, A. Nasri, R. Schober, R. K. Mallik, and V. K. Bhargava, "Network coded cooperative BICM-OFDM," in Proc. IEEE Global Communications Conference (GLOBECOM) 2011, Houston, Texas, USA, pp. 1-6, Dec. 2011

[27] M. Iezzi, M. Di Renzo, and F. Graziosi, "Network code design from unequal error protection coding: channel-aware receiver design and diversity analysis", in Proc. IEEE International Conference on Communications (ICC) 2011, Kyoto, Japan, pp.1-6, Jun. 2011

[28] D. Chase, "Code combining-A maximum-likelihood decoding approach for combining an arbitrary number of noisy packets," IEEE Transactions on Communications, vol. 33, no. 5, pp. 385-393, May 1985

[29] L. Zheng and D. Tse, "Diversity and multiplexing: a fundamental tradeoff in multiple-antenna channels," IEEE Transactions on Information Theory, vol. 49, no. 5, pp. 1073-1096, May 2003

[30] Y. Li, Q. Yin, W. Xu, and H. Wang, "On the design of relay selection strategies in regenerative cooperative networks with outdated CSI", IEEE Transactions on Wireless Communnications, vol. 10, no. 9, pp. 30863097, Sept. 2011 
This article has been accepted for publication in a future issue of this journal, but has not been fully edited. Content may change prior to final publication.

[31] G.C. Alexandropoulos, A. Papadogiannis, and P.C. Sofotasios, "A comparative study of relaying schemes with decode and forward over Nakagami-m fading channels," Journal of Computer Networks and Communications, vol. 2011, Article ID 560528, pp. 1-14, 2011.

[32] F. Xu, F. Lau, Q. Zhou, and D. Yue, "Outage performance of cooperative communication systems using opportunistic relaying and selection combining receiver," IEEE Signal Processing Letters, vol. 16, no. 4 , pp. 237-240, Apr. 2009

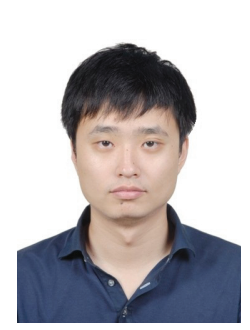

Zhang Zhang received the B.Eng. and Ph.D. degrees from Beijing University of Posts and Telecommunications (BUPT), Beijing, China, in 2007 and 2012, respectively. From May 2009 to June 2012, he also served as a Research Assistant for the Wireless and Mobile Communications Technology R\&D Center, Tsinghua University, Beijing, China. $\mathrm{He}$ is currently with the Department of Research and Innovation, Alcatel Lucent Shanghai Bell, Shanghai, China.

His current research interests include cooperative communication, network coding, machine type communication and network information theory.

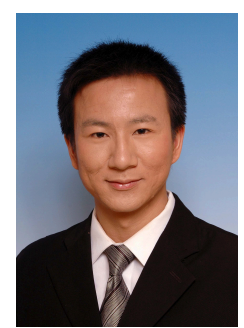

Shaoshi Yang (S'09) received the B.Eng. Degree in information engineering from Beijing University of Posts and Telecommunications, Beijing, China, in 2006. He is currently working toward the Ph.D. degree in wireless communications with the School of Electronics and Computer Science, University of Southampton, Southampton, U.K., through scholarships from both the University of Southampton and the China Scholarship Council.

From November 2008 to February 2009, he was an Intern Research Fellow with the Communications Technology Laboratory, Intel Labs China, Beijing, where he focused on Channel Quality Indicator Channel design for mobile WiMAX (802.16 m). His research interests include multiuser detection/multiple-input mutliple-output detection, multicell joint/distributed processing, cooperative communications, green radio, and interference management. He has published in excess of 20 research papers on IEEE journals and conferences.

Shaoshi is a recipient of the PMC-Sierra Telecommunications Technology Scholarship, and a Junior Member of the Isaac Newton Institute for Mathematical Sciences, Cambridge, UK. He is also a TPC member of both the 23rd Annual IEEE International Symposium on Personal, Indoor and Mobile Radio Communications (IEEE PIMRC 2012), and of the 48th Annual IEEE International Conference on Communications (IEEE ICC 2013).

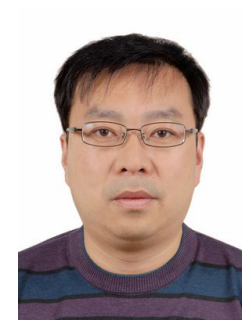

Tiejun Lv (M'08-SM'12) received the M.S. and $\mathrm{Ph} . \mathrm{D}$. degrees in electronic engineering from the University of Electronic Science and Technology of China (UESTC), Chengdu, China, in 1997 and 2000 , respectively. From January 2001 to December 2002, he was a Postdoctoral Fellow with Tsinghua University, Beijing, China. From September 2008 to March 2009, he was a Visiting Professor with the Department of Electrical Engineering, Stanford University, Stanford, CA. He is currently a Full Professor with the School of Information and Communication Engineering, Beijing University of Posts and Telecommunications (BUPT). He is the author of more than 100 published technical papers on the physical layer of wireless mobile communications. His current research interests include signal processing, communications theory and networking.

Dr. Lv is also a Senior Member of the Chinese Electronics Association. He was the recipient of the Program for New Century Excellent Talents in University Award from the Ministry of Education, China, in 2006.

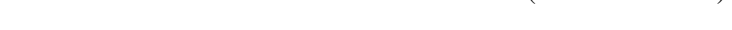

